\title{
Reconstructing Tree-Child Networks from Reticulate-Edge-Deleted Subnetworks
}

\section{Yukihiro Murakami $^{1}$ (D) - Leo van lersel ${ }^{1} \cdot$ Remie Janssen $^{1} \cdot$ Mark Jones $^{1}$. Vincent Moulton ${ }^{2}$}

Received: 5 February 2019 / Accepted: 3 July 2019 / Published online: 11 July 2019

(c) The Author(s) 2019

\begin{abstract}
Network reconstruction lies at the heart of phylogenetic research. Two well-studied classes of phylogenetic networks include tree-child networks and level- $k$ networks. In a tree-child network, every non-leaf node has a child that is a tree node or a leaf. In a level- $k$ network, the maximum number of reticulations contained in a biconnected component is $k$. Here, we show that level- $k$ tree-child networks are encoded by their reticulate-edge-deleted subnetworks, which are subnetworks obtained by deleting a single reticulation edge, if $k \geq 2$. Following this, we provide a polynomial-time algorithm for uniquely reconstructing such networks from their reticulate-edge-deleted subnetworks. Moreover, we show that this can even be done when considering subnetworks obtained by deleting one reticulation edge from each biconnected component with $k$ reticulations.
\end{abstract}

Keywords Phylogenetic network · Network encoding · Tree-child networks · Reticulate-edge-deleted subnetworks

Yukihiro Murakami

yukimurakami07201994@gmail.com

Leo van Iersel

1.j.j.v.iersel@gmail.com

Remie Janssen

remiejanssen@gmail.com

Mark Jones

markelliotlloyd@gmail.com

Vincent Moulton

V.Moulton@uea.ac.uk

1 Delft Institute of Applied Mathematics, Delft University of Technology, Van Mourik

Broekmanweg 6, 2628 XE Delft, The Netherlands

2 School of Computing Sciences, University of East Anglia, Norwich NR4 7TJ, UK 


\section{Introduction}

Phylogenetic trees are instrumental in representing the evolutionary history of a set of species $X$. Leaves (extant species) are bijectively labeled by $X$, and speciation events are depicted by internal nodes (non-extant species). Though powerful in their own right, phylogenetic trees are limited by their inability to display complex evolutionary events such as horizontal gene transfers, hybridizations, and recombinations (Sneath 1975). For such reticulate (non-treelike) events, there has been increased interest in employing phylogenetic networks instead, which are generalizations of phylogenetic trees to directed acyclic graphs (Morrison 2005; Huson et al. 2010).

In recent years, heavy focus has been cast upon the reconstruction of phylogenetic networks. Many existing methods of tree reconstruction such as maximum parsimony, maximum likelihood, and distance-based methods have been adapted to network reconstruction (Hein 1990; von Haeseler and Churchill 1993; Strimmer and Moulton 2000; Jin et al. 2006; Bordewich et al. 2018b; Huson et al. 2010). In this paper, we tackle the reconstruction problem through a building block approach. Building blocks are generally some class of subnetworks, e.g., binets (van Iersel et al. 2017), trinets (Huber and Moulton 2013), or trees, used to infer the original network. A potential problem here is that there could be more than one network with the same building blocks. When considering trees as building blocks, Pardi and Scornavacca somewhat resolved this distinguishability issue by considering 'canonical forms' of networks; however, the problem still persists in general (Pardi and Scornavacca 2015). Therefore, the goal in any building block approach is to see if it encodes the network. We say that a network is encoded by a certain building block if given two networks containing the same set of this building block, the networks are isomorphic.

It has been shown by Huber et al. (2014) that there exist networks which are not encoded by all subnetworks (called subnets) induced on proper subsets of the taxa. This is not to say that subnets do not encode many networks; in fact, it has been shown time and time again that considering topologically restricted classes of networks can help bypass this complication (Willson 2011; van Iersel and Moulton 2014; Gambette et al. 2017; van Iersel et al. 2017). Two of the more prominent network classes are the tree-child networks (Cardona et al. 2009) and the level-k networks (Jansson and Sung 2006). In a tree-child network, every non-leaf node has a child that is a tree node (nodes with indegree-1 and outdegree-2) or a leaf (nodes with indegree-1 and outdegree-0). In a level- $k$ network, the maximum number of reticulations (nodes with indegree-2 and outdegree-1) in a biconnected component (blob) is $k$ (see Fig. 1 for an example of a level-4 tree-child network).

In this paper, we show that binary level- $k$ tree-child networks, where $k \geq 2$ are encoded by reticulate-edge-deleted subnetworks, which are subnetworks obtained by deleting a single reticulation edge. In fact, we prove an even stronger result that this network class is encoded by its Maximum Lower-Level Subnetworks (MLLSs), the subnetworks obtained by deleting a reticulation edge from every level- $k$ biconnected component. We do so by exploiting the fact that tree-child networks contain either a cherry or a reticulated cherry (Bordewich and Semple 2016). Cherries need not be reconstructed, since they stay intact in every MLLS; therefore we focus on reconstructing reticulated cherries and show that they are uniquely reconstructible through 
Fig. 1 (Color figure online) A level-4 tree-child network $N$ on the set of species $X=$ $\{a, \ldots, n\}$. Though $N$ is a directed acyclic graph, the edge directions are omitted to avoid cluttering. The arcs are directed downwards. The leaf pair $\{e, f\}$ is a cherry since they share a common parent. The leaf pair $\{a, b\}$ is a reticulated cherry since the parent of $a$ is also the parent of the parent of $b$
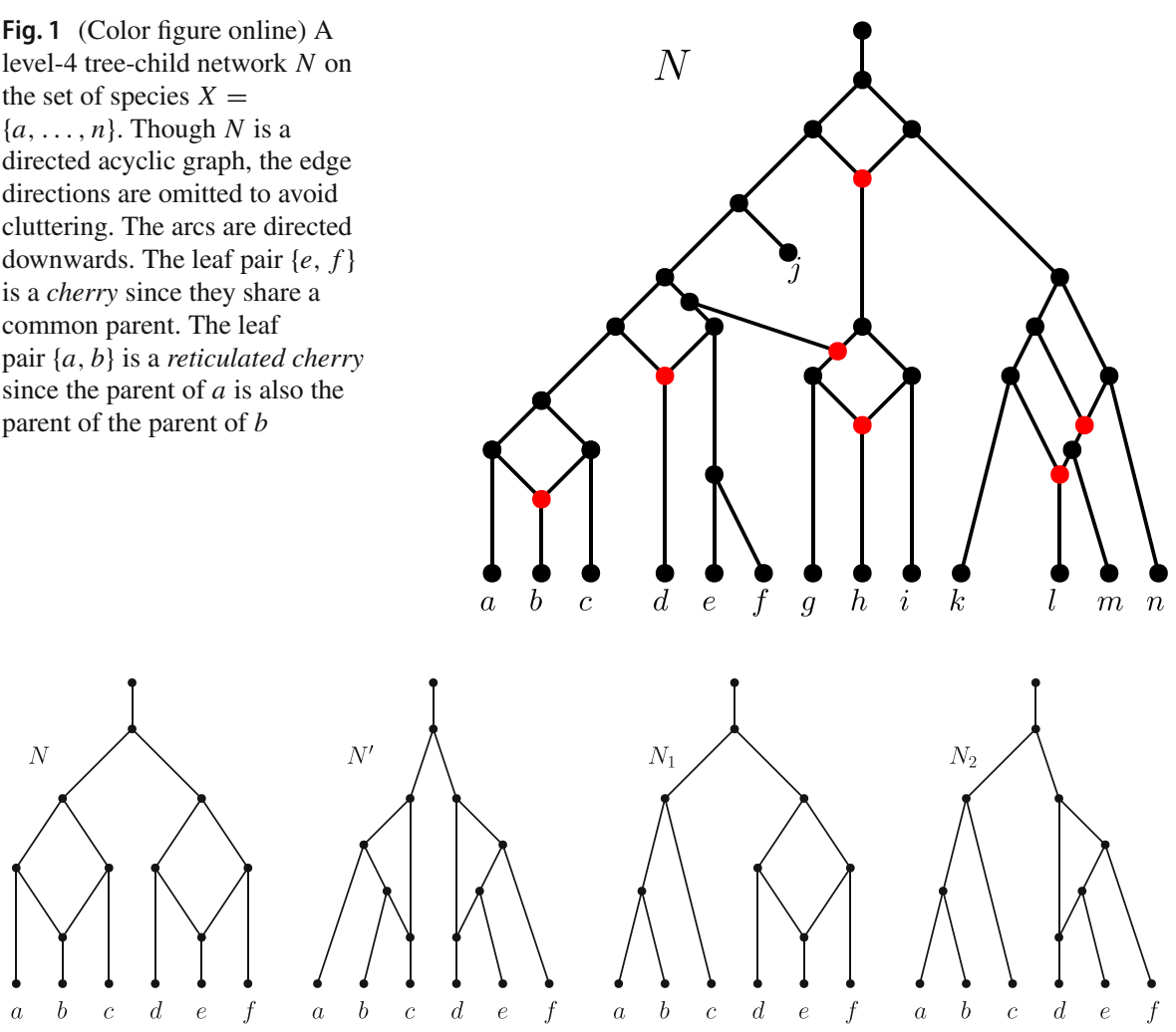

Fig. 2 Networks $N$ and $N^{\prime}$ are non-isomorphic but have the same lower-level subnetworks. Hence, any class containing $N$ and $N^{\prime}$ is not level-reconstructible. However, these networks have different subnetworks: $N_{1}$ is a subnetwork of $N$ but not of $N^{\prime} ; N_{2}$ is a subnetwork of $N^{\prime}$ but not of $N$. So $\left\{N, N^{\prime}\right\}$ is subnetworkreconstructible

an exhaustive case study. In proving this result, we explore 'blob trees', an underlying tree of a network, introduced initially by Gusfield and Bansal (2005). These labeled trees are obtained from networks by collapsing every biconnected component to a single node, labeling the node by its set of leaf-descendants, and removing the leaves. In this paper, we introduce the class of valid networks; for such a class, we show that we can reconstruct the blob tree of the original network from the blob trees of all MLLSs (Theorem 3). The class of tree-child networks are contained within the class of valid networks, and therefore, the result also follows for tree-child networks.

In the related literature, it has been shown that tree-child networks are encoded by trinets (van Iersel and Moulton 2014) but not by trees (see Fig. 2). Gambette et al. (2017) showed that level-1 networks (which are necessarily tree-child) with girth (shortest cycle in underlying graph) at least 5 are reconstructible from their triplets. The triplets are phylogenetic trees on 3 leaves; as the set of triplets can be computed from the set of all displayed trees of the network, level-1 networks of girth at least 5 are encoded by trees and therefore by their MLLSs. Others have also constructed level-1 networks (also called gt-networks) from trees. Nakhleh et al. showed that it 
was possible to find a level-1 network with the minimum number of reticulations that displays an input of two binary trees in polynomial time if such a network exists (Nakhleh et al. 2005). In the same paper, they also considered the following problem, which we restate using our notation. Given an input of two non-binary trees, find a level-1 network $N$ with one reticulation such that $N$ displays two MLLSs that are refinements of the two input non-binary trees, if such a network exists. Huynh et al. (2005) generalized this result by showing that one can find a level-1 network with the minimal number of reticulations for an input size of at least two non-binary trees, if such a network exists. They did not, however, consider whether the output network was unique, which is our focus for this paper. In particular, we focus on showing that certain networks are uniquely defined by their MLLSs and that they can be uniquely reconstructed from them; this is fundamentally different from the problem of finding a most parsimonious network for a set of trees - which, coincidentally, is a subset of MLLSs for a level-1 network.

The paper is organized as follows. In the next section, we define essential terms relevant to this paper, including MLLSs and the notion of encoding / reconstructibility. Section 3 presents the definitions and the key results on blob trees. In Sect. 4, we investigate the possible topologies for each leaf pair. Per our definition, there are 5 possibilities for each leaf pair up to isomorphism, and we develop a method for reconstructing a blob containing a particular leaf pair topology. In Sect. 5, we show our main result for this paper, that binary level- $k \geq 2$ tree-child networks are reconstructible from their MLLSs (Theorem 6). A polynomial-time (in the size of the leaf set and the MLLS set) algorithm for reconstructing tree-child networks from their MLLSs follows naturally from our proof, and we present this in Sect. 6. In the last section, we conclude with some discussion of potential future directions.

\section{Preliminaries and Definitions}

Definition 1 Let $X$ be a non-empty finite set. A rooted binary phylogenetic network $N$ on $X$ is a directed acyclic graph (a directed graph with no directed cycles) in which every node is in one of the following categories:

1. one node of indegree-0 and outdegree-1 (the root);

2. $|X|$ nodes of indegree-1 and outdegree-0 (leaf nodes or leaves);

3. nodes of indegree-1 and outdegree-2 (tree nodes); and

4. nodes of indegree-2 and outdegree-1 (reticulations).

The leaves are bijectively labeled with label set $X$, where the leaf set is sometimes denoted $L(N)$.

We will henceforth refer to rooted binary phylogenetic networks as networks. The edges feeding into reticulations are called reticulation edges, and each non-reticulation edge is called a tree edge. We write $v \in N$ to denote that $v$ is a node in $N$. Given an edge $(x, y)$ in $N$, we say that $x$ is a parent of $y$ and $y$ is a child of $x$. A directed path of length $n$ from $x$ to $y$ is a sequence of edges $\left(v_{0}, v_{1}\right), \ldots,\left(v_{n-1}, v_{n}\right)$ such that $x=v_{0}, y=v_{n}$, where $v_{i}$ is a parent of $v_{i+1}$ for $i=0, \ldots, n-1$. The node $x$ is an ancestor of / above $y$, or $y$ is a descendant of / below $x$ if there is a directed path 
from $x$ to $y$ in $N$. Two nodes are incomparable if neither nodes are above the other. The network $N$ is tree-child if every non-leaf node in $N$ is a parent of a tree node or a leaf. A tree path is a directed path that contains no reticulations except possibly for its starting node. It is easy to see that, for each node $v$ of a tree-child network, there exists a tree path to a leaf.

Two networks $N, N^{\prime}$ on $X$ are isomorphic if there exists a bijection $f$ between the vertices of $N$ and the vertices of $N^{\prime}$ such that $(u, v)$ is an edge of $N$ if and only if $(f(u), f(v))$ is an edge of $N^{\prime}$ and each leaf of $N$ is mapped to a leaf of $N^{\prime}$ with the same label.

Definition 2 Deleting a node $x$ from a network is the action of removing $x$ and all of its incident edges from $N$. Deleting an edge $(x, y)$ from a network is the action of removing $(x, y)$ from $N$.

A cut-node is a node of a network whose deletion disconnects the network. A cut-edge is an edge of a network whose deletion disconnects the network. A pendant subnetwork of a network $N$ is obtained by deleting a cut-edge $(x, y)$ from $N$ and taking the connected component containing $y$. A pendant subtree is a pendant subnetwork that is a tree.

Definition 3 A biconnected component of a network $N$ is a maximal subgraph with at least three nodes such that no node of the subgraph is a cut-node of the subgraph. A blob is either a biconnected component or a tree node that is not in a biconnected component.

We say $N$ is a level- $k$ network, denoted $l v l(N)=k$, if the maximum number of reticulations contained in any biconnected component is $k$ (Jansson and Sung 2006). A level-0 network is a tree (a network with no reticulations). Since the level of a blob is the number of reticulations it contains, a tree node that is not in a biconnected component is a level-0 blob.

We say that a network $N$ on $X$ displays a network $N^{\prime}$ on $X$ if some subgraph $N^{\prime \prime}$ of $N$ is a subdivision of $N^{\prime}$ (i.e., if $N^{\prime \prime}$ can be obtained from $N^{\prime}$ by replacing directed edges by directed paths). An alternative view of when a network is displayed by another network is based on cleaning up a directed acyclic graph.

Definition 4 Cleaning up a directed acyclic graph is the act of applying the following operations until none is applicable:

1. delete an unlabeled outdegree- 0 node;

2. suppress an indegree-1 outdegree-1 node (i.e., if $(u, v),(v, w)$ are edges in a graph where $v$ is an indegree- 1 outdegree- 1 vertex, we suppress $v$ by deleting the node $v$ and adding an edge $(u, w)$.);

3. replace a pair of parallel edges by a single edge, i.e., delete one of the parallel edges and suppress both the parent node and the child node.

Note that cleaning up a directed acyclic graph, obtained from a network on $X$ by deleting, for each reticulation, at most one of the incoming reticulation edges, returns a network on $X$. 
Lemma 1 If a network $N$ on $X$ displays a network $N^{\prime}$ on $X$, then we can obtain $N^{\prime}$ from $N$ by deleting, for each reticulation, at most one of the two incoming reticulation edges, and subsequently cleaning up.

Proof Since $N$ displays $N^{\prime}$, some subgraph of $N$ is a subdivision of $N^{\prime}$. Because of this, there is an embedding of $N^{\prime}$ into $N$ where the nodes and edges of $N^{\prime}$ are mapped to nodes and paths of $N$, such that these paths are edge disjoint. Without loss of generality, this embedding contains the root of $N$. For each reticulation of which exactly one incoming reticulation edge is used by the embedding, delete the other incoming reticulation edge, and subsequently clean up the directed acyclic graph. We claim that all unused edges in the embedding have been removed in the resultant network $M$.

Suppose not. Then, there exists an edge in $M$ that is not used in the embedding of $N^{\prime}$ into $N$. Consider a lowest such edge $(x, y)$.

Node $y$ cannot be a leaf of $N$ because all leaves of $N$ are in the embedding of $N^{\prime}$ into $N$.

Now suppose that $y$ is a tree node of $N$. It is not possible that an outgoing edge of $y$ is in the embedding, because the root of the embedding is the root of $N$. Hence, the outgoing edges of $y$ are not in the embedding. At least one of these outgoing edges of $y$ is in $M$ because otherwise $y$ would have been deleted by cleaning up rule 1 . Hence, at least one outgoing edge of $y$ is in $M$ but not in the embedding of $N^{\prime}$ into $N$, contradicting the assumption that $(x, y)$ is a lowest such edge.

Hence, $y$ is a reticulation. If the other incoming edge of the reticulation is also not in the embedding, it follows similarly to the previous case that the outgoing edge of $y$ is in $M$ but not in the embedding, contradicting the assumption that $(x, y)$ is a lowest such edge. Hence, exactly one incoming edge of $y$ is used by the embedding. Therefore, the other incoming edge, $(x, y)$, has been deleted, contradicting the assumption that $(x, y)$ is an edge of $M$.

This implies that every edge in $M$ is used in the embedding of $N^{\prime}$ into $N$. Since in addition all indegree-1 outdegree-1 nodes have been suppressed by cleaning up rule 2, we have that $M$ is $N^{\prime}$.

Let $\mathcal{N}(N)$ denote the set of all networks on $X$ that are displayed by a network $N$ on $X$, excluding $N$ itself. The networks in $\mathcal{N}(N)$ are called the subnetworks of $N$. A class $\mathcal{C}$ of networks is called subnetwork-reconstructible if for any two networks $N, N^{\prime} \in \mathcal{C}$ with $\mathcal{N}(N)=\mathcal{N}\left(N^{\prime}\right)$, we have that $N$ and $N^{\prime}$ are isomorphic.

A related but subtly different notion is the following. Let $N$ be a level- $k$ network. Then, $\mathcal{N}^{k-1}(N)$ denotes the set of subnetworks of $N$ that are of level at most $k-1$. The networks in $\mathcal{N}^{k-1}(N)$ are called the lower-level subnetworks of $N$. Then, a class $\mathcal{C}$ of networks is called level-reconstructible if for any two networks $N, N^{\prime} \in \mathcal{C}$ of level- $k$ and $\mathcal{N}^{k-1}(N)=\mathcal{N}^{k-1}\left(N^{\prime}\right)$, we have that $N$ and $N^{\prime}$ are isomorphic. Note that if a network is level-reconstructible, then it is subnetwork-reconstructible. The converse is not true in general, and an example of this is shown in Fig. 2.

In this paper, we prove a result that is stronger than level-reconstructibility. We first define a type of reticulation edge deletion, and we introduce a corresponding subclass of networks. 
Definition 5 A reticulation edge deletion is valid if the resulting subnetwork, after cleaning up, contains exactly 2 nodes and 3 edges fewer than the original network, i.e., only the reticulation edge is deleted and its endpoints suppressed. A reticulation edge deletion is invalid otherwise. Call a reticulation edge valid/invalid if its deletion is valid / invalid.

Definition 6 Networks are valid if all reticulation edges in the network are valid.

An example of a valid reticulation edge is shown in Fig. 3.

\section{Lemma 2 All reticulation edges in a tree-child network are valid.}

Proof Let $N$ be a tree-child network and suppose for a contradiction that deleting some reticulation edge $e=(u, v)$ is invalid. We note that $v$ is a reticulation. As $N$ is tree-child, we also have that $u$ is a tree node. Therefore, after deleting $e, u$ and $v$ will each be indegree- 1 outdegree- 1 nodes and will be suppressed by cleaning up. This removes a total of 2 nodes and 3 edges. Hence, to show that $e$ is valid, it remains to show that no further cleaning up occurs after deleting $e$ and suppressing $u$ and $v$. As all remaining nodes have the same indegree and outdegree as before, there are no unlabeled outdegree 0 nodes and no remaining indegree- 1 outdegree- 1 nodes. So we just need to show that deleting $e$ creates no parallel edges.

We split the proof into three sub-cases. First assume that suppressing $u$ results in the creation of parallel edges. Then, we must have that $u$ is contained in a 'triangle' with nodes $x, y$ and edges $(x, u),(x, y),(u, y)$. But then $y$ is a reticulation, implying that $u$ is the parent of two reticulations $y$ and $v$. Thus, $u$ has no child that is a tree node or a leaf, contradicting the tree-child property of $N$. Next assume that suppressing $v$ results in the creation of parallel edges. Then, we must have that $v$ is contained in a triangle with nodes $x, y$ and edges $(x, v),(x, y),(v, y)$. But then $y$ is a reticulation, implying that $v$ is the parent of a reticulation $y$. Thus, $v$ has no child that is a tree node or a leaf, contradicting the tree-child property of $N$. Finally, assume that suppressing both $u$ and $v$ results in the creation of parallel edges. Then, we must have that $e$ formed the central edge of a 'diamond' with nodes $x, y$ and edges $(x, u),(x, v),(u, v),(u, y),(v, y)$. However, this cannot occur since the child of $v, y$, would be a reticulation, which again contradicts the tree-child property of $N$.

Therefore, every reticulation edge of a tree-child network is valid.

The above lemma does not hold for general networks (see Fig. 3). Intuitively, Lemma 2 states that removing any reticulation edge from a tree-child network is self-contained, and it does not affect any other reticulations within the network. No additional information is 'lost' when deleting valid reticulation edges. In particular, Lemma 2 implies that tree-child networks are valid.

From here onwards, it is implicitly assumed that the network $N^{\prime}$ obtained by deleting some reticulation edges from $N$ undergoes cleaning up.

Definition 7 A maximum subnetwork of a network $N$ is a subnetwork obtained by a single reticulation edge deletion from $N$.

Lemma 3 Every maximum subnetwork of a tree-child network is tree-child. 

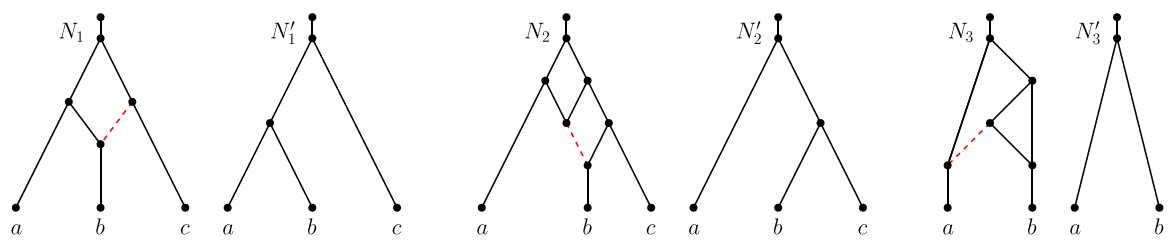

Fig. 3 (Color figure online) Three networks $N_{1}, N_{2}, N_{3}$ with their respective maximum subnetworks $N_{1}^{\prime}, N_{2}^{\prime}, N_{3}^{\prime}$ obtained by deleting the red reticulation edge and subsequently cleaning up. The red reticulation edge in $N_{1}$ is valid; however, the red dashed reticulation edges in $N_{2}$ and $N_{3}$ are invalid. The subnetwork $N_{2}^{\prime}$ contains 4 fewer nodes and 6 fewer edges than $N_{2}$, and $N_{3}^{\prime}$ contains 3 fewer nodes and 5 fewer edges than $N_{3}$
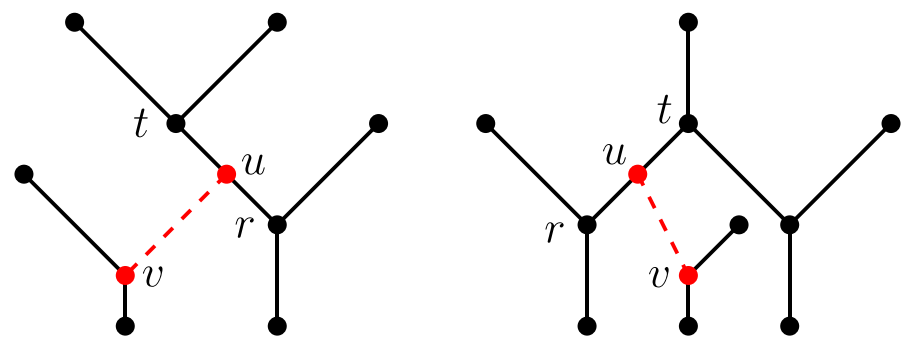

Fig. 4 (Color figure online) Visual aid for the proof of Lemma 3. The left case is when $N^{\prime}$ contains a reticulation $t$ that is a parent of a reticulation $r$. The right case is when a tree node $t$ is a parent of two reticulations. In either case, the red dashed edge $(u, v)$ must be inserted in these particular places to obtain $N$, and in either case $N$ is not tree-child

Proof Suppose that there exists a tree-child network $N$ with a maximum subnetwork $N^{\prime}$ that is not tree-child. Then, there exists a node $t$ in $N^{\prime}$ such that all of its children are reticulations. Let $(u, v)$ be the reticulation edge deleted from $N$ to obtain $N^{\prime}$. Since $t$ has a tree node as a child in $N$, node $u$ must be a child of $t$ in $N$. Hence, $(t, u)$ and $(u, r)$ are edges in $N$, for some child $r$ of $t$ in $N^{\prime}$. But then $N$ is not tree-child as $u$ is the parent of only reticulations $v$ and $r$, a contradiction (see Fig. 4).

Definition 8 For $k \geq 1$, a maximum lower-level subnetwork (MLLS) of a level- $k$ network $N$ is a subnetwork obtained by deleting exactly one valid reticulation edge from every level- $k$ blob in $N$. Let $\mathcal{N}^{m l l s}(N)$ denote the set of all MLLSs of $N$.

Observe that as long as $\mathcal{N}^{m l l s}(N)$ is a non-empty set, it is equal to the set of all subnetworks of $N$ with level at most $k-1$ and a maximum number of edges.

By considering each reticulation edge deletion separately, it follows from Lemma 3 that the MLLSs of a tree-child network are tree-child.

A class $\mathcal{C}$ of networks is called MLLS-reconstructible if for any two networks $N, N^{\prime} \in \mathcal{C}$ with $\mathcal{N}^{m l l s}(N)=\mathcal{N}^{m l l s}\left(N^{\prime}\right)$, we have that $N$ and $N^{\prime}$ are isomorphic. Because all MLLSs are lower-level subnetworks of $N$, we have $\mathcal{N}^{m l l s}(N) \subseteq$ $\mathcal{N}^{k-1}(N)$. Therefore, if a class of networks is MLLS-reconstructible, then it is levelreconstructible. The converse also holds for valid networks. 
Lemma 4 Let $N$ be a level-k valid network. Then, we may obtain $\mathcal{N}^{k-1}(N)$ from $\mathcal{N}^{m l l s}(N)$.

Proof Let $M \in \mathcal{N}^{k-1}(N)$. As $M$ is a lower-level subnetwork of $N$, by Lemma $1, M$ must have been obtained from $N$ by deleting at least one reticulation edge, say $e_{i}$, from every level- $k$ blob, say $B_{i}$, in $N$, and deleting some reticulation edges from other blobs. By definition of MLLSs, there must exist an MLLS $N^{\prime} \in \mathcal{N}^{m l l s}(N)$ that was obtained from $N$ by deleting $e_{i}$ from $B_{i}$. Then clearly, some subnetwork of $N^{\prime}$, obtained by deleting the rest of the reticulation edges, is $M$. That is, $\mathcal{N}^{k-1}(N)$ is precisely the set of all subnetworks of the networks of $\mathcal{N}^{m l l s}(N)$, and the networks of $\mathcal{N}^{m l l s}(N)$ (i.e., $\left.\mathcal{N}^{k-1}(N)=\mathcal{N}^{m l l s}(N) \cup \bigcup_{M \in \mathcal{N}^{m l l s}(N)} \mathcal{N}(M)\right)$.

Corollary 1 Given a class $\mathcal{C}$ of networks containing only valid networks, if the class is level-reconstructible, then it is MLLS-reconstructible.

Proof Let $N, N^{\prime} \in \mathcal{C}$ with $\mathcal{N}^{m l l s}(N)=\mathcal{N}^{m l l s}\left(N^{\prime}\right)$. By Lemma 4, this implies that $\mathcal{N}^{k-1}(N)=\mathcal{N}^{k-1}\left(N^{\prime}\right)$. As the class is level-reconstructible, we have that $N$ and $N^{\prime}$ must be isomorphic.

Note that this result does not hold in general, as networks may contain invalid reticulation edges that cannot be deleted to obtain an MLLS.

Observation 1 Let $\mathcal{C}$ be a class of networks. If $\mathcal{C}$ is $M L L S$-reconstructible, then $\mathcal{C}$ is also level-reconstructible. If $\mathcal{C}$ is level-reconstructible, then $\mathcal{C}$ is also subnetworkreconstructible.

We will henceforth assume that all considered networks are binary tree-child networks on a non-empty set of taxa $X$, unless stated otherwise.

\section{Blob Trees}

In this section, we show how to reconstruct a blob tree, the underlying tree of a network. The tree has a similar construction as the 'blobbed trees' in Gusfield and Bansal (2005) with further modifications.

Definition 9 The blob tree of a network $N$, denoted $B T(N)$, is the labeled tree obtained by applying the following:

1. contract every blob into a single node, and label each node, except for the root node, by the leaf-descendant set of the top node of the blob;

2. delete all leaf nodes.

We call the nodes in $B T(N)$ blob nodes.

An example of a blob tree is illustrated in Fig. 5.

We refer to the top nodes of blobs as pure nodes. In the case of a level-0 blob, this top node is simply the tree node itself. Let $x$ denote the pure node of some blob $B$ of some network $N$. Then $\operatorname{desc}_{N}(x)=\operatorname{desc}_{N}(B)$ denotes the set of leaf-descendants of $x$ (and thus $B$ ) in $N$. 

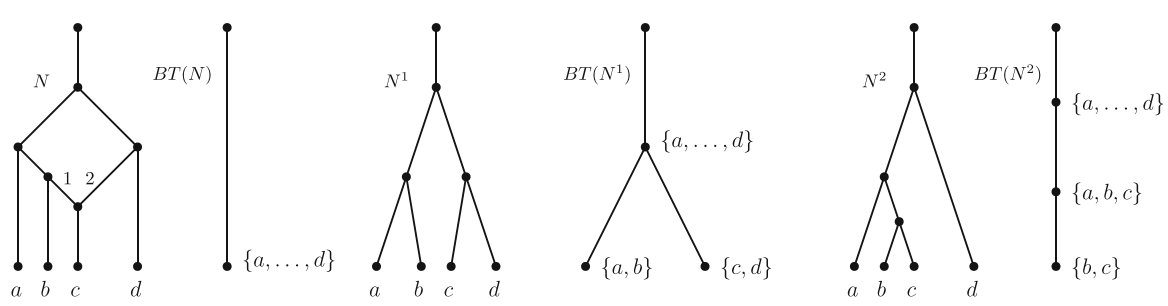

Fig. 5 A tree-child network $N$, its maximum subnetworks $N^{1}, N^{2}$ obtained from deleting edges 1 and 2 , respectively, together with their blob trees

For a general network $N$, it is possible for $B T(N)$ to contain two nodes with the same label if there is a blob in $N$ of indegree- 1 and outdegree-1. However, the same cannot occur in tree-child networks, due to the following lemma.

Lemma 5 Let $N$ be a tree-child network on $X$, let $A \subseteq X$ and let $x$ be a highest tree

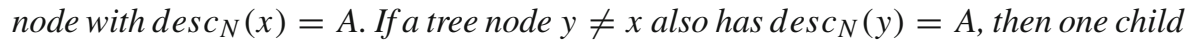
of $x$ is a reticulation $r$ such that $y$ is below $x$ and $y$ is above $r$. Hence, $x$ is the unique highest tree node with $\operatorname{desc}_{N}(x)=A$ and all other tree nodes $y$ with $\operatorname{desc}_{N}(y)=A$ are in the same blob.

Proof Let $y \neq x$ be a tree node with $\operatorname{desc}_{N}(y)=A$. To begin, note that $y$ must be either above or below $x$. To see this, note that by the tree-child property of $N$, there exists a leaf $l$ that is reached by $x$ via a tree path. Then, for $y$ to be an ancestor of $l, y$ must be either above or below $x$. Hence, $x$ is the unique highest tree node with $\operatorname{desc}_{N}(x)=A$ and $y$ is below $x$.

By the tree-child property of $N$, either $x$ can have two children that are tree nodes or leaves, or $x$ can have one tree node or leaf child and one reticulation child. Let $c_{1}, c_{2}$ denote the children of $x$, and by the tree-child property of $N$, there exist leaves $l_{1}, l_{2}$ that are reached by $c_{1}, c_{2}$ via tree paths, respectively.

First suppose that the two children $c_{1}, c_{2}$ of $x$ are tree nodes or leaves. Then, for $y$ to be an ancestor of both $l_{1}$ and $l_{2}, y$ must be an ancestor of both $c_{1}$ and $c_{2}$, contradicting that $y \neq x$ is below $x$.

Hence, one of the two children of $x$ is a reticulation $r$. Without loss of generality, $r=$ $c_{1}$. It remains to show that $y$ is above $c_{1}$. Since $y$ is an ancestor of $l_{1}$, and there is a tree path from $c_{1}$ to $l_{1}$, node $y$ is either above or below $c_{1}$. Suppose for contradiction that $y$ is below $c_{1}$. Since $y$ is also an ancestor of $l_{2}$, there exists a directed path from $y$ to $l_{2}$. This path must pass through $x$ since the path from $x$ to $l_{2}$ is a tree path. This is a directed path from $y$ to $x$. However, since there is also a directed path from $x$ to $y$ (via $c_{1}$ ), and $y \neq x$, it follows that there exists a directed cycle, a contradiction.

The following corollary follows immediately from Lemma 5.

Corollary 2 Let $N$ be a tree-child network. Then, its blob tree BT $(N)$ contains nodes with unique labels.

Due to this, we identify blob nodes by their node labels, e.g., for a blob $B$ in $N$ with $\operatorname{desc}_{N}(B)=A$, the corresponding blob node in $B T(N)$ is $A$. 


\subsection{On Reticulated Cherries}

Let $x$ and $y$ be two non-reticulation nodes in a network $N$. Let $p_{x}, p_{y}$ be the parents of $x, y$, respectively, where $p_{y}$ is a reticulation and $p_{x}$ is a parent of $p_{y}$. Let $g_{y}$ denote the parent of $p_{y}$ that is not $p_{x}$ (see Fig. 6a). We call the subgraph of $N$ induced by the nodes $x, y, p_{x}, p_{y}$, and $g_{y}$ a reticulated cherry shape. We will refer to the reticulated cherry shape as $\langle x, y\rangle$ and say that the nodes $x$ and $y$ form the reticulated cherry shape. In this case, we say that the reticulation is on $y$ and that the reticulation $p_{y}$ is in the reticulated cherry shape $\langle x, y\rangle$. This notion is a generalization of the reticulated cherries defined by Bordewich et al. (2018b), in which both $x$ and $y$ are leaves.

Lemma 6 In a tree-child network, all reticulations are in a reticulated cherry shape. Moreover, for $k \geq 1$, there is at least one reticulation in each level-k blob that is in a reticulated cherry shape formed by two nodes outside of the blob.

Proof Let $N$ be a tree-child network and consider a reticulation $r$ in an arbitrarily chosen blob $B$. By the tree-child property, $r$ must have a non-reticulation child $y$ and two tree node parents $t_{1}, t_{2}$. The child of $t_{1}$ that is not $r$ must be a non-reticulation $x$. Then, $r$ is in a reticulated cherry shape formed by $x$ and $y$.

Now consider a lowest tree node $a$ in $B$. If both children of $a$ were to be non-reticulations then at least one of the children would also be contained in $B$, contradicting our choice of $a$. If both children of $a$ were to be reticulations, then the network would no longer be tree-child, a contradiction. Thus, one child of $a$ is a reticulation, say $c$, and the other a non-reticulation, say $x$. The child of $c$, say $y$, must be a non-reticulation as the network is tree-child, and thus, $B$ contains a reticulated cherry shape formed by two nodes $x, y$. Moreover, $x$ and $y$ are outside of $B$ because they are either leaves or tree nodes, and below a lowest tree node in $B$.

A reticulated cherry shape $\langle x, y\rangle$ is called a lowest reticulated cherry shape of a blob $B$, if the parent $p_{x}$ of $x$ is a lowest tree node of $B$. This implies that $x$ and $y$ are not contained in $B$, as shown in the proof of Lemma 6 .

Suppose we are given a reticulated cherry shape $\langle x, y\rangle$ with the reticulation on $y$ and let $g_{y}$ be the parent of $p_{y}$ that is not $p_{x}$. We use the following operations defined by Bordewich et al. (2018b).

- cutting $\langle x, y\rangle$ is the operation of deleting $\left(p_{x}, p_{y}\right)$ and suppressing $p_{x}$ and $p_{y}$.

- isolating $\langle x, y\rangle$ is the operation of deleting $\left(g_{y}, p_{y}\right)$ and suppressing $g_{y}$ and $p_{y}$.

Let $N^{\prime}$ be a maximum subnetwork of a tree-child network $N$ obtained by isolating a lowest reticulated cherry shape of a blob $B$. Then, there is a pure node in $N^{\prime}$ that is not a pure node in $N$ (Fig. 6). Moreover, if blob $B$ is of level at least 2, the leaf-descendant set of the new pure node is not equal to the leaf-descendant set of any node in $N$. This leads to the following observation.

Observation 2 For a tree-child network $N$ and $B$ a level-k blob, with $k \geq 2$, there is always a reticulation edge we can delete from $B$ such that the blob tree of the resulting subnetwork is not equal to $B T(N)$. 


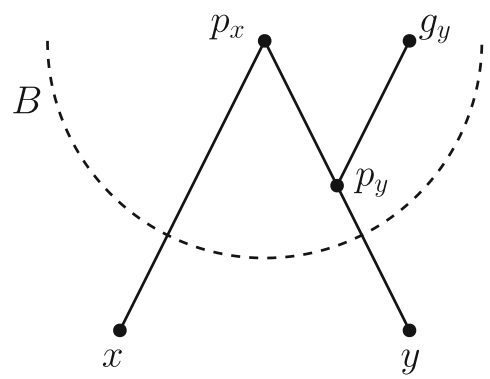

(a)

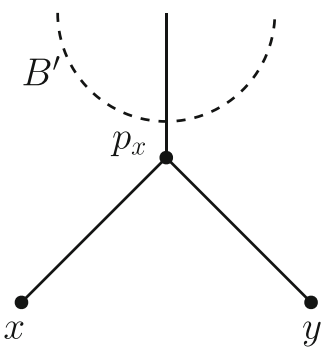

(b)

Fig. 6 a A portion of the network showing a lowest reticulated cherry shape in a blob $B$. b The same portion of the network after isolating the reticulate cherry shape $\langle x, y\rangle$. Note here that $p_{x}$ is a pure node in the subnetwork, but $p_{x}$ is not a pure node in the original network

Now suppose $x$ and $y$ are both leaves. If $x$ and $y$ share a common parent, then they form a cherry. If $x$ and $y$ form a reticulated cherry shape, then it is a reticulated cherry. The following Lemma from Bordewich and Semple (2016) is essential for our results and will be used extensively throughout the text.

Lemma 7 (Bordewich and Semple 2016) If $N$ is a tree-child network on at least two leaves, then $N$ contains either a cherry or a reticulated cherry.

\subsection{Reconstructing the Blob Tree of a Tree-Child Network}

Lemma 8 For a valid network $N$, if the blob tree $B T(N)$ contains a blob node $A$, then $B T\left(N^{\prime}\right)$ contains the blob node A for every maximum subnetwork $N^{\prime}$ of $N$.

Proof First suppose that $A$ is a blob node corresponding to a level-0 blob in $N$. The corresponding node $t$ in $N$ is not incident to any reticulation edges, so it is not possible to suppress $t$ via a reticulation edge deletion. Note that a reticulation edge deletion from a blob above or below $t$ would not change the leaf-descendant set of $t$. Hence, $t$ remains a level-0 blob in all maximum subnetworks of $N$ with leaf-descendant set $A$. Thus, $A$ is a blob node in all $B T\left(N^{\prime}\right)$ for all maximum subnetworks $N^{\prime}$ of $N$.

Now suppose that $A$ is a blob node corresponding to a blob of level at least 1 . Suppose $t$ is the corresponding pure node in $N$. If $t$ is not incident to a reticulation edge, then there is no way of suppressing $t$ by means of edge deletions and any reticulation edge deletion will not change the leaf-descendant set of $t$. Hence, $t$ is a pure node with leaf-descendant set $A$ in all maximum subnetworks of $N$. If, on the other hand, there is a reticulation $r$ with edges $(t, r),(s, r)$, then let $c$ be the child of $t$ that is a tree node (it is possible that $c=s$ ). Because $t$ is the top node of the blob, there is a directed path from $t$ to $s$, which must include $c$. Hence, there is a directed path from $c$ to $s$ and to $r$. Therefore, we have $\operatorname{desc}_{N}(r) \subseteq \operatorname{desc}_{N}(c)$. $\operatorname{Sodesc}_{N}(c)=\operatorname{desc}_{N}(c) \cup \operatorname{desc}_{N}(r)=\operatorname{desc}_{N}(t)=A$. We now use the fact that after a valid edge deletion, only the endpoints of the edge are suppressed in the resultant maximum subnetwork. The maximum subnetwork where $(t, r)$ is deleted contains $c$ as a pure node, and hence, $A$ is a blob node in its blob tree. The maximum subnetwork 
where $(s, r)$ is deleted contains $t$ as a pure node, and hence, $A$ is a blob node in its blob tree. The maximum subnetwork where some other reticulation edge is deleted contains $t$ as a pure node, and hence, $A$ is a blob node in its blob tree. Thus, $A$ is a blob node in $B T\left(N^{\prime}\right)$ for all maximum subnetworks $N^{\prime}$ of $N$.

Lemma 9 For a validnetwork $N$, if $B T\left(N^{\prime}\right)$ contains a blob node A for every maximum subnetwork $N^{\prime}$ of $N$, then $B T(N)$ also contains the blob node $A$.

Proof Consider some lowest reticulation $r$ in $N$ such that $r$ is the ancestor of some $a \in A$. Let $c$ be the child of $r$ in $N$. Since $r$ is of outdegree-1, we have $\operatorname{desc}_{N}(r)=$ $\operatorname{desc}_{N}(c)$. We may assume $\operatorname{desc}_{N}(r) \neq A$, as otherwise $c$ is the root of a pendant subtree spanning $A$ in $N$, and consequently $A$ is a blob node in $B T(N)$. Let $(u, r),(v, r)$ be the edges leading into $r$. Let $N^{\prime}, N^{\prime \prime}$ be the maximum subnetworks of $N$ obtained by deleting $(u, r),(v, r)$, respectively. Note here that every node $x$ in $N^{\prime}$ or $N^{\prime \prime}$ is also a node in $N$. We now examine the relations between $\operatorname{desc}_{N}(r)$ and $A$ exhaustively.

- Suppose $\operatorname{desc}_{N}(r) \not \subset A$ and $A \not \subset \operatorname{desc}_{N}(r)$. We show that there is no node in $N^{\prime}$ that has leaf-descendant set $A$. By assumption, there exists a node $a^{\prime} \in \operatorname{desc}_{N}(r)$ such that $a^{\prime} \notin A$. Then, $a^{\prime} \in \operatorname{desc}_{N^{\prime}}(c)$. Let $x$ be a node in $N^{\prime}$ (which is also a node in $N$ ). We examine the relations between $x$ and $c$ in $N^{\prime}$ exhaustively.

- If $x$ is an ancestor of $c$ in $N^{\prime}$ then $\operatorname{desc}_{N^{\prime}}(x) \neq A$ since $a^{\prime} \in \operatorname{desc}_{N^{\prime}}(c) \subseteq$ $\operatorname{desc}_{N^{\prime}}(x)$.

- If $x$ is a descendant of $c$ in $N^{\prime}$ then $\operatorname{desc}_{N^{\prime}}(x) \neq A$ since $A \not \subset \operatorname{desc}_{N^{\prime}}(c)$.

- If $x$ is incomparable to $c$ in $N^{\prime}$ then $\operatorname{desc}_{N^{\prime}}(x) \neq A$ since $a \notin \operatorname{desc}_{N^{\prime}}(x)$ by assumption that $r$ was the lowest reticulation above $a$.

It follows that $A$ is not in $B T\left(N^{\prime}\right)$, and this case is not possible. The only possibilities then are either $A \subsetneq \operatorname{desc}_{N}(r)$ or $\operatorname{desc}_{N}(r) \subsetneq A$.

By assumption, $B T\left(N^{\prime}\right)$ and $B T\left(N^{\prime \prime}\right)$ both contain $A$. Because of this, there are corresponding pure nodes $x^{\prime}, x^{\prime \prime}$ in $N^{\prime}, N^{\prime \prime}$ (also in $N$ ), respectively, with $\operatorname{desc}_{N^{\prime}}\left(x^{\prime}\right)=$ $\operatorname{desc}_{N^{\prime \prime}}\left(x^{\prime \prime}\right)=A$.

- Suppose $A \subsetneq \operatorname{desc}_{N}(r)$. Then, $x^{\prime}$ must be a descendant of $c$ in $N^{\prime}$, implying that $x^{\prime}$ must be a descendant of $r$ in $N$. We claim that $x^{\prime}$ is a pure node in $N$ with $\operatorname{desc}_{N}\left(x^{\prime}\right)=A$. If $x^{\prime}$ is not a pure node in $N$ then there exists a reticulation $s \neq$ $r$ below $x^{\prime}$ where $s$ and $x^{\prime}$ are contained in the same blob in which $x^{\prime}$ is not the top node, in $N$. The edge deletion does not suppress or delete the node $s$, since $s$ is a descendant of $r$, and any directed path from $r$ to $s$ is of length at least 2. Then, $s$ is a reticulation that is below $r$ such that the leaf-descendant set of $s$ contains an element of $A$. This contradicts our choice of $r$, so $x^{\prime}$ must be a pure node in $N$. Furthermore, we must have $\operatorname{desc}_{N}\left(x^{\prime}\right)=\operatorname{desc}_{N^{\prime}}\left(x^{\prime}\right)=A$ where the first equality holds as deleting a reticulation edge from above a node does not change its leafdescendant set in the resultant subnetwork. Then, $x^{\prime}$ must be a pure node in $N$ with $\operatorname{desc}_{N}\left(x^{\prime}\right)=A$ and we are done.

- So we may assume $\operatorname{desc}_{N}(r) \subsetneq A$. We now claim that $\operatorname{desc}_{N}(v) \subseteq A$. Suppose not. Noting that $v$ is not suppressed in $N^{\prime}$ (since $N$ is a valid network), and 
since $\operatorname{desc}_{N^{\prime}}(v)=\operatorname{desc}_{N}(v)$, we split into the three possible cases for the relation between $x^{\prime}$ and $v$ in $N^{\prime}$.

- If $x^{\prime}$ is an ancestor of $v$, then it is also an ancestor of $b \notin A$ in $N^{\prime}$ for some $b \in$ $\operatorname{desc}_{N^{\prime}}(v)$, a contradiction.

- If $x^{\prime}$ is incomparable to $v$, then $x^{\prime}$ is also incomparable to $c$ in $N^{\prime}$. Then, since $a \in A$ is a leaf-descendant of $x^{\prime}$ in $N^{\prime}$, there is a reticulation $s$ below $r$ in $N$ such that $s$ is an ancestor of $a$, which contradicts our choice of $r$.

- If $x^{\prime}$ is a descendant of $v$, then it must either be incomparable to or be a descendant of $c$ in $N^{\prime}$.

- If $x^{\prime}$ is incomparable to $c$ in $N^{\prime}$, then we reach a contradiction by the same argument as above.

- If $x^{\prime}$ is a descendant of $c$ in $N^{\prime}$, then as $\operatorname{desc}_{N^{\prime}}(c) \subsetneq A\left(\right.$ since $\operatorname{desc}_{N}(r)=$ $\operatorname{desc}_{N^{\prime}}(c)$ ) we have that $\operatorname{desc}_{N^{\prime}}\left(x^{\prime}\right) \subsetneq A$, a contradiction.

Thus, we have that $\operatorname{desc}_{N}(v) \subseteq A$. By an analogous reasoning on $x^{\prime \prime}$ in $N^{\prime \prime}$, we have that $\operatorname{desc}_{N}(u) \subseteq A$. It follows that $x^{\prime}$ must be an ancestor of $v$ in $N^{\prime}$, and so $x^{\prime}$ must be an ancestor of $v$ in $N$. It also follows that $x^{\prime}$ must be an ancestor of $u$ in $N$ to ensure that there is a path from $x^{\prime}$ to the leaf-descendants of $u$ in $N^{\prime}$.

We now claim that $x^{\prime}$ is also a pure node in $N$ with leaf-descendant set $A$. Indeed, adding the edge $(u, r)$ to $N^{\prime}$ (after undoing any cleaning up) only joins descendants of $x^{\prime}$, implying $x^{\prime}$ has leaf-descendant set $A$ in $N$. Furthermore, it cannot add any nodes that are not descended from $x^{\prime}$ to the blob containing $x^{\prime}$. It follows that $x^{\prime}$ remains a pure node in $N$ with leaf-descendant set $A$.

By combining the previous two lemmas, we see that the blob trees of valid networks are reconstructible from their maximum subnetworks.

Theorem 1 For a valid network $N$, given a set $A \subseteq X$, the blob tree $B T(N)$ contains the blob node $A$ if and only if $B T\left(N^{\prime}\right)$ contains the blob node A for every maximum subnetwork $N^{\prime}$ of $N$.

Proof Follows from Lemmas 8 and 9.

We can prove a similar result for MLLSs.

Theorem 2 Let $N$ be a level- $k$ valid network, with $k \geq 1$. Given a set $A \subseteq X$, the blob tree $B T(N)$ contains the blob node $A$ if and only if $B T\left(N^{m l l s}\right)$ contains $A$ for every MLLS $N^{\text {mlls }}$ of $N$.

Proof Suppose first that the blob tree $B T(N)$ contains the node $A$, and let $N^{m l l s}$ be an MLLS of $N$ obtained by deleting the edges in the set $E=\left\{e_{1}, \ldots, e_{m}\right\}$. Consider the maximum subnetwork $N^{\prime}$ of $N$ obtained by deleting the reticulation edge $e_{1}$. By Theorem 1, $B T\left(N^{\prime}\right)$ contains the blob node $A$. Now consider the maximum subnetwork $N^{\prime \prime}$ of $N^{\prime}$ obtained by deleting the reticulation edge $e_{2}$. Then, $B T\left(N^{\prime \prime}\right)$ contains the blob node $A$ by Theorem 1. Continuing in this fashion for all edges in $E$ shows that $B T\left(N^{m l l s}\right)$ contains the blob node $A$. 
Now suppose that $A$ is not a blob node of $B T(N)$. We prove that then there exists an MLLS $N^{m l l s}$ of $N$ such that $B T\left(N^{m l l s}\right)$ does not contain the blob node $A$. Let $B$ denote the blob in $N$ with leaf-descendant set $D$, such that $D$ is the smallest set that contains $A$. Consequently, if there exists a pure node in an MLLS $N^{\text {mlls }}$ of $N$ with leaf-descendant set $A$, then it must be a node that was originally in the blob $B$. Now observe that deleting reticulation edges from blobs that are not $B$ do not affect the leaf-descendant set of nodes in $B$. Then, we may assume, without loss of generality, that $N$ is a single blob network. But then by Theorem 1, A is not a blob node in $B T\left(N^{m l l s}\right)$, for some MLLS $N^{m l l s}$ of $N$.

We call a set $A \subseteq X$ a foundation node of $N$ if $B T(N)$ contains the node $A$. Let $\mathcal{F}(N)$ be the set of all foundation nodes of $N$.

Theorem 3 For a level- $k$ valid network $N$, with $k \geq 1$, its blob tree $B T(N)$ is reconstructible from its MLLSs.

Proof By Theorem 2, the set of all foundation nodes $\mathcal{F}(N)$ consists of the blob nodes that appear in $B T\left(N^{m l l s}\right)$ for every MLLS $N^{m l l s}$ of $N$.

Then, the blob tree $B T(N)$ is the tree with vertex set $\mathcal{F}(N)$ and an edge $(A, B)$ precisely if $B \subsetneq A$ and there is no $C \in \mathcal{F}(N)$ with $B \subsetneq C \subsetneq A$.

\subsection{Minimum Number of MLLSs to Reconstruct the Blob Tree of a Tree-Child Network}

We consider the minimum number of MLLSs required to reconstruct the blob tree of a tree-child network. Let $r$ be some reticulation in a blob $B$. We call a node $s$ a pseudo pure node of $r$ if it is a lowest node in $B$ such that there are two edge disjoint directed paths from $s$ to $r$.

Lemma 10 Let $N$ be a level-k tree-child network where $k \geq 1$. Two maximum subnetworks $N^{\prime}$ and $N^{\prime \prime}$ of $N$ suffice to reconstruct $B T(N)$.

Proof Let $r$ be a lowest reticulation in some blob $B$. Let $\langle x, y\rangle$ denote the reticulated cherry shape that contains $r$. Let $p_{x}$ and $p_{y}=r$ be the parents of $x$ and $y$, respectively, and let $g_{y}$ be the parent of $p_{y}$ that is not $p_{x}$. Let $N^{\prime}$ and $N^{\prime \prime}$ be the maximum subnetworks of $N$ derived by cutting and isolating $\langle x, y\rangle$, respectively. Let $F^{\prime}$ and $F^{\prime \prime}$ denote the set of foundation nodes of $N^{\prime}$ and $N^{\prime \prime}$, respectively, that are not foundation nodes of $N$. We claim that the intersection of $F^{\prime}$ and $F^{\prime \prime}$ is empty, from which it follows that the intersection of the node sets of $B T\left(N^{\prime}\right)$ and $B T\left(N^{\prime \prime}\right)$ contains the foundation nodes of $N$. Since by Lemma 8 each foundation node of $N$ is a foundation node of each maximum subnetwork, it follows that the intersection of the node sets of $B T\left(N^{\prime}\right)$ and $B T\left(N^{\prime \prime}\right)$ is precisely the set of all foundation nodes of $N$.

Let $P^{\prime}$ denote the set of all pure nodes in $N^{\prime}$ that have leaf-descendant sets in $F^{\prime}$. Similarly let $P^{\prime \prime}$ denote the set of all pure nodes in $N^{\prime \prime}$ that have leaf-descendant sets in $F^{\prime \prime}$. We prove the following claims regarding the pure nodes of $P^{\prime}$ and $P^{\prime \prime}$.

Claim 1 Let $p \in P^{\prime}\left(p \in P^{\prime \prime}\right)$. Then $p$ is an ancestor of $r$ in $N$. 
Proof 1 Suppose not. First suppose that $p$ is a descendant of $r$ in $N$. As $r$ is a lowest reticulation in $N, p$ is a tree node or a leaf in $N^{\prime}\left(N^{\prime \prime}\right)$. If $p$ is a tree node, then $p$ must have been a pure node in $N$ to begin with: the pendant subnetwork rooted at the child of $r$ is an invariant upon obtaining maximum subnetworks of $N$, since $r$ is a lowest reticulation. This contradicts the fact that $p$ is an element of $P^{\prime}\left(P^{\prime \prime}\right)$. If $p$ is a leaf, then $p$ cannot be a pure node in $N^{\prime}\left(N^{\prime \prime}\right)$, a contradiction.

Now suppose that $p$ is incomparable to $r$ in $N$. Let $p$ be the pure node of a blob $B^{\prime}$ in $N^{\prime}\left(B^{\prime \prime}\right.$ in $\left.N^{\prime \prime}\right)$. As $p$ is not an ancestor of $r$ in $N, p$ must also not be an ancestor of $p_{x}$ nor $g_{y}$ in $N$. We see that $B^{\prime}\left(B^{\prime \prime}\right)$ remains a blob after adding the edge $\left(p_{x}, r\right)$ to $N^{\prime}\left(\left(g_{y}, r\right)\right.$ to $\left.N^{\prime \prime}\right)$, and so $p$ remains a pure node in $N$, a contradiction.

Claim 2 Let $p \in P^{\prime}\left(p \in P^{\prime \prime}\right)$, and let $s$ be a pseudo pure node of $r$ in $N$. Then, $p$ is a descendant of $s$ in $N$ and $p \neq s$.

\section{Proof 2 Suppose not.}

If $p$ is equal to or strictly above $s$ then $p$ is an ancestor of both $p_{x}$ and $g_{y}$. Adding the edge $\left(p_{x}, r\right)$ to $N^{\prime}\left(\left(g_{y}, r\right)\right.$ to $\left.N^{\prime \prime}\right)$ only joins descendants of $p$. Furthermore, it cannot add any nodes that are not descended from $p$ to the blob in $N^{\prime}\left(N^{\prime \prime}\right)$ containing $p$. It follows that $p$ remains a pure node in $N$, a contradiction.

Now suppose that $p$ is incomparable to $s$. If $p$ is not in the blob $B$, then as reticulation edge deletions do not affect other blobs, we have that $p$ must have been a pure node in $N$. This contradicts our assumption on $p$. So $p$ must be in the blob $B$. Since $p$ is incomparable to $s$, but $p$ must still be an ancestor of $r$ by Claim $1, p$ must be an ancestor of a reticulation $r^{\prime}$ such that $s$ is an ancestor of $r^{\prime}$ and $r^{\prime}$ is an ancestor of $r$. The parent of $s$, denoted $p_{s}$, is not suppressed in both $N^{\prime}$ and $N^{\prime \prime}$. Now $p_{s}$ is either above or incomparable to $p$, and the two nodes belong to the blob which contains $r^{\prime}$ in $N^{\prime}\left(N^{\prime \prime}\right)$ (see Fig. 7a). It follows that $p$ cannot be a pure node in $N^{\prime}\left(N^{\prime \prime}\right)$, which contradicts our assumption.

It remains to show that $F^{\prime} \cap F^{\prime \prime}=\emptyset$. Let $P_{x}^{\prime}=\left\{p \in P^{\prime}: p\right.$ is an ancestor of $p_{x}$ in $N\}$ and let $P_{y}^{\prime}=\left\{p \in P^{\prime}: p\right.$ is an ancestor of $g_{y}$ in $\left.N\right\}$. By Claim 1, we have $P^{\prime}=P_{x}^{\prime} \cup P_{y}^{\prime}$. By Claim 2, we have $P_{x}^{\prime} \cap P_{y}^{\prime}=\emptyset$. Let $P_{x}^{\prime \prime}=\left\{p \in P^{\prime \prime}\right.$ : $p$ is an ancestor of $p_{x}$ in $\left.N\right\}$ and let $P_{y}^{\prime \prime}=\left\{p \in P^{\prime \prime}: p\right.$ is an ancestor of $g_{y}$ in $\left.N\right\}$. Similarly, we have $P^{\prime \prime}=P_{x}^{\prime \prime} \cup P_{y}^{\prime \prime}$ and $P_{x}^{\prime \prime} \cap P_{y}^{\prime \prime}=\emptyset$. Let $a^{\prime} \in P_{x}^{\prime}, a^{\prime \prime} \in P_{x}^{\prime \prime}$, and $b^{\prime \prime} \in P_{y}^{\prime \prime}$. Let $u \in \operatorname{desc}_{N}(x)$ and let $v \in \operatorname{desc}_{N}(y)$. Clearly, $u \in \operatorname{desc}_{N^{\prime}}\left(a^{\prime}\right)$

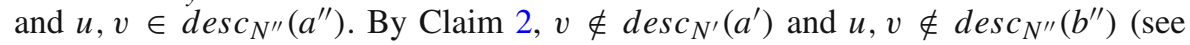
Fig. 7b). This implies that for some $A \in F^{\prime}$ such that $u \in A$, we have $A \notin F^{\prime \prime}$. An analogous argument shows that for some $B \in F^{\prime}$ such that $v \in B$, we have $B \notin F^{\prime \prime}$. Because of the way in which we defined the network $N^{\prime}$, all foundation nodes in $F^{\prime}$ must contain the element $u$ or $v$, but not both. Thus, the above two cases cover all foundation nodes in $F^{\prime}$; therefore, $F^{\prime}$ and $F^{\prime \prime}$ are disjoint.

Let $N$ be a network and let $N_{A}$ be a pendant subnetwork of $N$ rooted by a node with leaf-descendant set $A$. Collapsing $N_{A}$ from $N$ means that we replace $N_{A}$ by a leaf $A$. Let $N \backslash N_{A}$ denote the network obtained by collapsing $N_{A}$ from $N$.

Lemma 11 Let $N$ be a tree-child network, and let $N_{A}$ denote a pendant subnetwork of $N$ rooted at a node with leaf-descendant set A. Then, BT $\left(N \backslash N_{A}\right)$ is obtained from $B T(N)$ by deleting the pendant subtree rooted at $A$. 


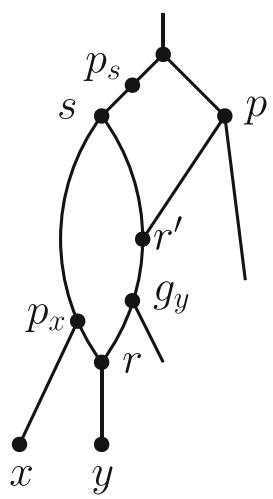

(a)

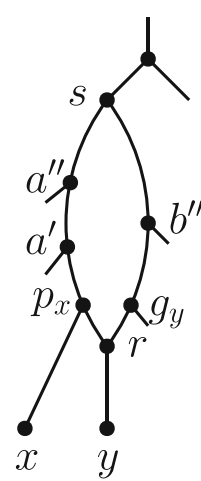

$N$

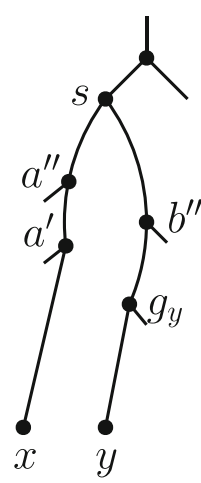

$N^{\prime}$

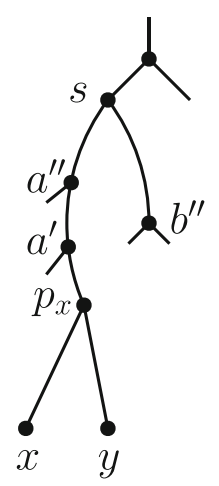

$N^{\prime \prime}$

(b)

Fig. 7 Visual aid for Lemma 10 proof. a Proof of Claim 2. Cutting or isolating $\langle x, y\rangle$ results in subnetworks where $p_{s}$ and $p$ lie in the blob containing $r^{\prime}$. b Proof of the paragraph after Claim 2, which shows that $P^{\prime} \cap$ $P^{\prime \prime}=\varnothing$

Proof By definition, there exists a blob node $A$ in $B T(N)$. Note that pendant subnetworks of $N$ uniquely correspond to a pendant subtree of $B T(N)$, by definition of blob trees and also because node labels of blob trees are unique for tree-child networks (Corollary 2). Then, the pendant subtree of $B T(N)$ rooted at $A$ is uniquely defined by $N_{A}$ and vice versa: this implies the lemma.

Lemma 12 Let $N$ be a level-k tree-child network with $k \geq 2$. Two MLLSs $N_{1}^{\text {mlls }}$ and $N_{2}^{\text {mlls }}$ of N suffice to reconstruct BT $(N)$. In particular, $N_{1}^{m}$ lls is the MLLS obtained by cutting a lowest reticulated cherry shape in every level-k blob, and $N_{2}^{m l l s}$ is the MLLS obtained by isolating these reticulated cherry shapes.

Proof We prove the lemma by induction on the number of level- $k$ blobs $l$ in $N$. For the base case, there is only one level- $k$ blob in $N$. By Lemma 10, we are done.

So suppose now that $N$ contains $l \geq 2$ level- $k$ blobs. Consider a lowest level- $k$ blob $B$ in $N$, and let $A$ denote the leaf-descendant set of $B$. Let $N_{A}$ and $N_{i A}^{m l l s}$ denote the pendant subnetwork of $N$ and $N_{i}^{\text {mlls }}$ rooted at the pure node with leaf-descendant set $A$, for $i=1,2$. By Theorem 2, $A$ is a blob node in $B T\left(N_{i}^{m l l s}\right)$ for $i=1,2$, and therefore, such pendant subnetworks exist. Note that the pendant subnetworks $N_{i A}^{m l l s}$ are maximum subnetworks of $N_{A}$ obtained by cutting and isolating the reticulated cherry shape associated with some lowest reticulation $r$. By Lemma 10, we have that $N_{1 A}^{m l l s}$ and $N_{2 A}^{m l l s}$ suffice to reconstruct $B T\left(N_{A}\right)$. We now collapse $N_{i A}^{m l l s}$ from the MLLS $N_{i}^{m l l s}$ for $i=1,2$. Furthermore, we collapse $N_{A}$ from the network $N$. Note that $N \backslash N_{A}$ is a level- $k$ tree-child network with $l-1$ level- $k$ blobs, and that $N_{i}^{m l l s} \backslash N_{i A}^{m l l s}$ are MLLSs of $N \backslash N_{A}$ obtained by cutting and isolating a lowest reticulated cherry shape from every level- $k$ blob, for $i=1,2$, respectively. By the induction hypothesis, these two MLLSs of $N \backslash N_{A}$ suffice to reconstruct $B T\left(N \backslash N_{A}\right)$. Now by Lemma 11, we have that $B T(N)$ is the blob tree obtained by appending $B T\left(N_{A}\right)$ to $B T\left(N \backslash N_{A}\right)$. 
We append $B T\left(N_{A}\right)$ to the node $C$ in $B T\left(N \backslash N_{A}\right)$, such that $A \subseteq C$, and there exists no node $D \in B T\left(N \backslash N_{A}\right)$ where $A \subseteq D \subseteq C$.

Given $N, N_{1}^{m l l s}$, and $N_{2}^{m l l s}$ as in the setting of Lemma 12, the foundation nodes of $N$ can be found by taking the intersection of the foundation nodes of $N_{1}^{m l l s}$ and that of $N_{2}^{m l l s}$. Then, $B T(N)$ can be reconstructed as in the proof of Theorem 3 .

\subsection{Identifying the Level-k Blobs of a Tree-Child Network}

We now show that given the MLLSs, it is possible to identify which foundation nodes correspond to a level- $k$ blob in the original tree-child network.

Lemma 13 Let $N$ be a level-k tree-child network with $k \geq 2$. A blob of $N$ is level$k^{\prime}<k$ if and only if the set of children of the corresponding blob node in BT $\left(N^{m l l s}\right)$, for every $N^{\text {mlls }} \in \mathcal{N}^{m l l s}(N)$, is precisely the set of children of the blob node in $B T(N)$.

Proof Suppose $B$ is a level- $k^{\prime}<k$ blob in $N$. Then, $B$ remains intact (no reticulation edges deleted) in all MLLSs of $N$. Let $B^{\prime}$ be a blob in $N$ that is directly below $B$, and let $e$ denote the outgoing edge from $B$ to the pure node of $B^{\prime}$. The edge $e$ is not suppressed in any MLLS of $N$. And since edges are deleted to obtain MLLSs of $N$, we have that the number of leaves that are below the edge $e$ (below the child of $e$ ) stays the same since blobs are biconnected. By Theorem 2, every node in $B T(N)$ is a node in $B T\left(N^{m l l s}\right)$ for all MLLSs $N^{m l l s}$ of $N$. Furthermore for tree-child networks, the node labels in blob trees are unique. Then, the blob node of $B$ must have the blob node of $B^{\prime}$ as one of its children in the blob tree of all MLLSs. Since $B^{\prime}$ was chosen arbitrarily, this implies that the set of children of the blob node in $B T\left(N^{m l l s}\right)$, for every $N^{\text {mlls }} \in \mathcal{N}^{\text {mlls }}(N)$, is precisely the set of children of the blob node in $B T(N)$.

For the other direction, we prove the contrapositive. Suppose $B$ is a level- $k$ blob in $N$, and let $\operatorname{desc}_{N}(B)=A$. By Observation 2, we can isolate a lowest reticulated cherry in $B$ to obtain an MLLS $N^{m l l s}$ of $N$ where $B T\left(N^{m l l s}\right)$ is different from $B T(N)$. In this construction of $N^{m l l s}$, there exists a pure node in $N^{m l l s}$ which was not a pure node in $N$. Then, the set of children of $A$ in $B T\left(N^{m l l s}\right)$ is not the same as the set of children of $A$ in $B T(N)$.

Figure 8 illustrates Lemma 13 with a level-4 tree-child network $N$. The blob trees of its MLLSs are taken, from which the blob tree of $N$ can be reconstructed (Theorem 2). Then, it can be seen that the set of children of the blob node $\{a, \ldots, n\}$ in $B T\left(N_{i}\right)$ for $i=1,3,5,6,7,8$ differs from the set of children of $\{a, \ldots, n\}$ in $B T(N)$. Hence, the blob with leaf-descendant set $\{a, \ldots, n\}$ is of level-4. Since the children of the other blob nodes do not change, the blobs with leaf-descendant sets $\{a, b, c\},\{e, f\}$ and $\{k, \ldots, n\}$ are blobs of level lower than 4 .

\section{Leaf Pair Analysis}

In order to reconstruct a tree-child network from its MLLSs, we require a way of locating the position of the missing reticulation edges. In this section, we show that 

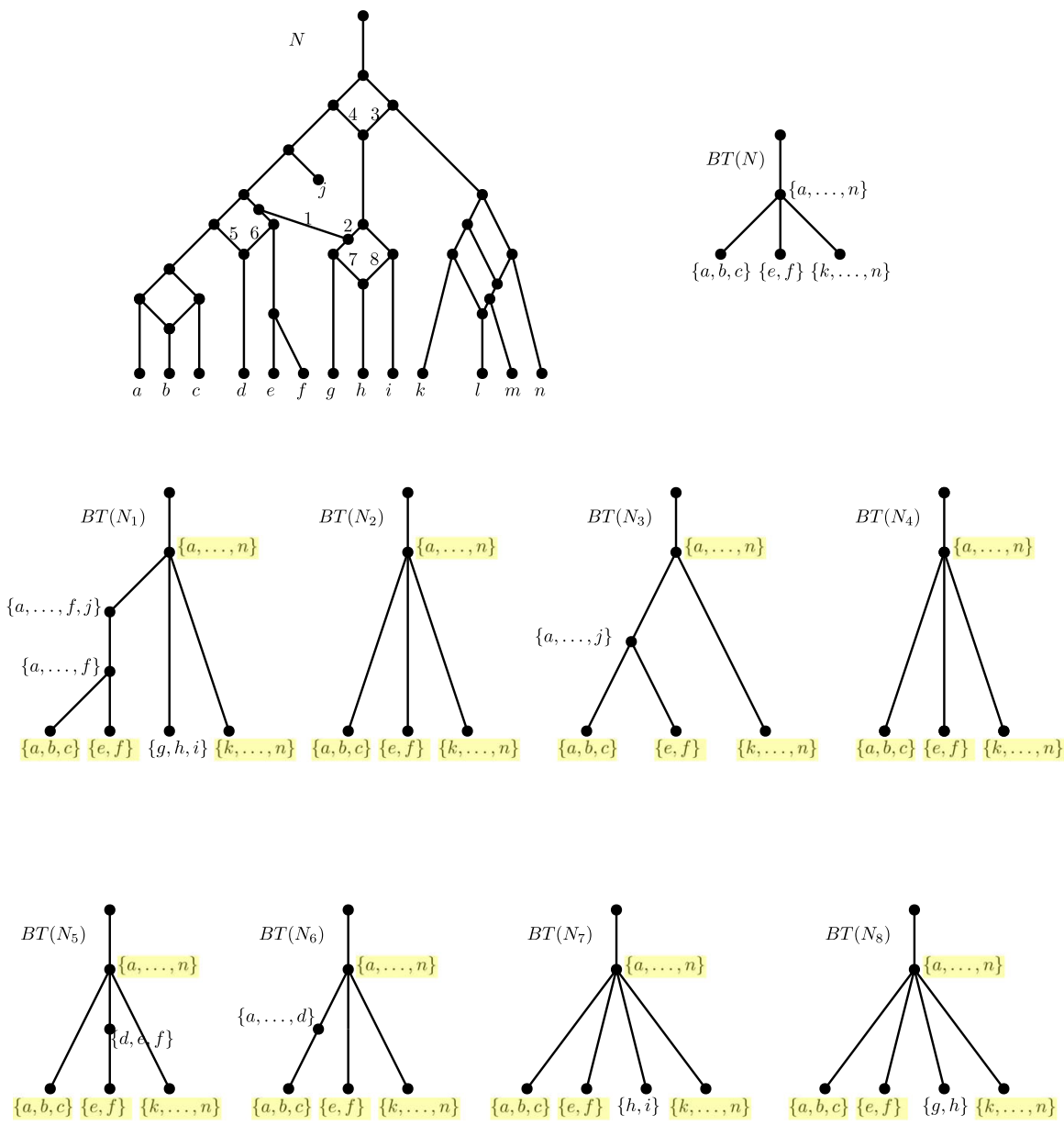

Fig. 8 (Color figure online) $N_{i}$ for $i=1,2, \ldots, 8$ refers to the MLLS of a level-4 tree-child network $N$ obtained by deleting the reticulation edge $i . B T(N)$ is the blob tree of the network $N$ and $B T\left(N_{i}\right)$ is the blob tree of $N_{i}$ for each $i$. The foundation nodes are highlighted in yellow

studying the topology of a leaf pair in the MLLSs gives enough information to infer the topology of those same leaves in the original network. The next section will show how we can use this to find the location of the missing reticulation edge of each blob by choosing the appropriate leaf pair.

We use the inter-node distance as defined by Bordewich and Semple (2016). For our purposes, we slightly tweak the definition by allowing the endpoints to be non-leaf nodes.

Definition 10 Let $N$ be a network and let $x, y \in N$. An up-down path of length $p$ from $x$ to $y$ is a sequence of nodes $x=v_{0}, v_{1}, v_{2}, \ldots, v_{p-1}, v_{p}=y$ in $N$, such that for some $0 \leq i \leq p, N$ contains the edges

$$
\left(v_{i}, v_{i-1}\right), \ldots,\left(v_{1}, x\right)
$$




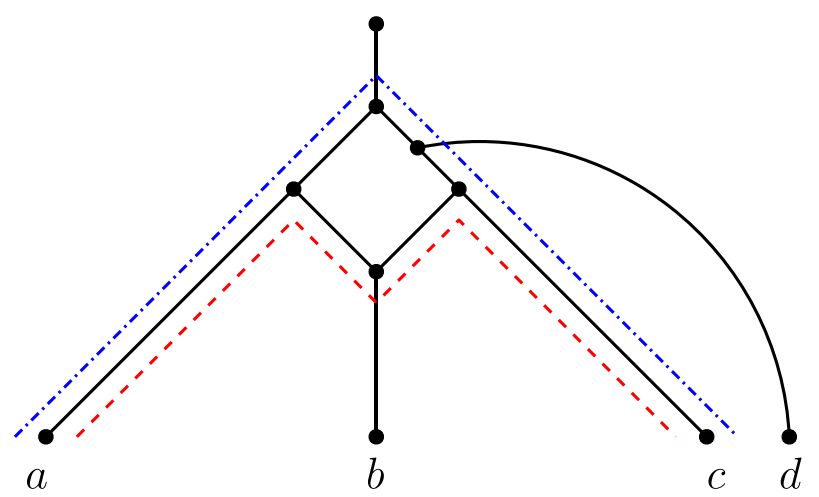

Fig. 9 (Color figure online) A network $N$ on 4 leaves. The shortest $a c$ up-down distance is 5 (blue dashdotted path); however, the shortest $a c$ distance in the underlying undirected graph of $N$ is 4 (red dashed path)

and

$$
\left(v_{i}, v_{i+1}\right),\left(v_{i+1}, v_{i+2}\right), \ldots,\left(v_{p-1}, y\right) .
$$

The node $v_{i}$ is the apex of this up-down path. The length of a shortest $x y$ up-down path $P$ in $N$ is denoted $d_{N}(x, y)$.

Note that the shortest up-down distance $d_{N}(x, y)$ in a network $N$ may not necessarily be the shortest distance in the underlying undirected graph of $N$ (where the underlying undirected graph of $N$ is obtained by replacing every directed edge by an undirected edge), see Fig. 9.

Let $Q$ be an up-down path between nodes $u$ and $v$ of length at least 2 in a tree-child network $N$. An edge $(u, v)$, if it exists, is called a shortcut. In some papers, the notion of a shortcut (also known as a redundant arc) is defined on directed paths rather than on up-down paths (Bordewich et al. 2018b; Willson 2010). For the purposes of this paper and since a directed path is by definition an up-down path (without the 'up' portion), we define shortcuts on the up-down paths. Call an up-down path which has no shortcuts in $N$ a shortcut free up-down path. Note that shortest up-down paths are necessarily shortcut free. Let $N^{\prime}$ be a maximum subnetwork of $N$ obtained by deleting some reticulation edge $(u, r)$. Let $P^{\prime}$ be an $x y$ up-down path in $N^{\prime}$ for nodes $x, y$. Reinsert the edge $(u, r)$ in $N^{\prime}$. Then, the $x y$ up-down path $P^{\prime}$, together with any nodes in $\{u, r\}$ that intersect some edge of $P^{\prime}$, is called the embedded path of $P^{\prime}$ in $N$.

Lemma 14 In a tree-child network, deleting a single reticulation edge can reduce the up-down distance between any two leaves by at most one.

Proof Let $N$ be a tree-child network and let $N^{\prime}$ be a maximum subnetwork of $N$ obtained by deleting some reticulation edge $(u, r)$. Let $v$ be the parent of $r$ in $N$ that is not $u$. Take any $x y$ up-down path $P^{\prime}$ in $N^{\prime}$, and let $P$ be its embedded path in $N$. Let $P^{*}$ be an up-down path in $N$ derived from $P$ by taking the shortcut $(u, r)$ if it is 


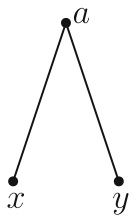

$\Lambda(x, y)$

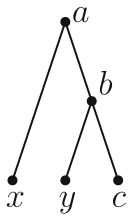

$\lambda(x, y)$

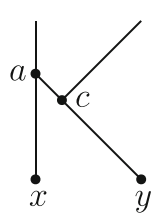

$K(x, y)$

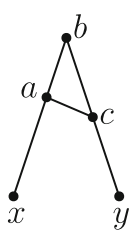

$A(x, y)$

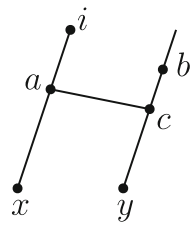

$H(x, y)$

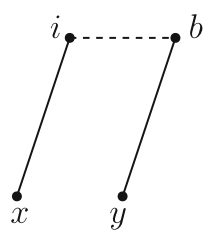

$\Pi(x, y)$

Fig. 10 All possible shapes on two leaves $\{x, y\}$ (up to permuting $x$ and $y$ ). The dashed line indicates that any $i b$ up-down path has length at least 2

a shortcut in $P$. We show that $\left|P^{*}\right| \leq\left|P^{\prime}\right|+1$. Now compared to $P^{\prime}$, the up-down path $P$ contains at most 2 additional nodes - the nodes $u$ and $r$. If it contains:

- 0 additional nodes then $(u, r)$ cannot be a shortcut of the embedded path $P$. So, $|P|=\left|P^{*}\right|=\left|P^{\prime}\right|$

- 1 additional node, then again, $(u, r)$ cannot be a shortcut of the embedded path $P$. So, $|P|=\left|P^{*}\right|=\left|P^{\prime}\right|+1$;

- 2 additional nodes, then $(u, r)$ must be a shortcut in $P$, as otherwise deleting $(u, r)$ disconnects $P^{\prime}$. This implies that currently, $P$ contains all three of the points $\{u, v, r\}$.

Then $\left|P^{\prime}\right|=|P|-2 \geq\left|P^{*}\right|-1$, where the inequality follows as taking a shortcut reduces the length of an up-down path by at least 1 .

It then follows that a single reticulation edge deletion from $N$ can reduce $d_{N}(x, y)$ for any two leaves $x, y \in N$ by at most 1 .

Lemma 15 Let $N$ be a tree-child network. For each pair of leaves $\{x, y\}$, exactly one of the following cases holds (see Fig. 10):

- $N$ contains a cherry $\Lambda(x, y)$ with nodes $a, x, y$ and edges $(a, x),(a, y)$;

- $N$ contains a cherry subdivided by one tree node. If this tree node is the parent of $y$, there is a subgraph with nodes $a, b, c, x, y$ and edges $(a, x),(a, b),(b, y),(b, c)$, which we call $\lambda(x, y)$;

- $N$ contains a reticulated cherry, which is a cherry subdivided by one reticulation. If this reticulation is the parent of $y$, there is a subgraph with nodes $a, b, c, i, x, y$ and edges $(a, x),(a, c),(b, c),(c, y),(i, a)$ which we call $K(x, y)$;

- if $i=b$, then we also call this shape $A(x, y)$;

- if $i \neq b$, then we also call this shape $H(x, y)$.

- if $d_{N}(x, y) \geq 4$, we say that $N$ contains $\Pi(x, y)$.

Hence, there are eight possibilities in total: $\Lambda(x, y), \lambda(x, y), \lambda(y, x), A(x, y)$, $A(y, x), H(x, y), H(y, x), \Pi(x, y)$, each of which we call a shape. However, keep in mind that when $N$ contains $\Pi(x, y)$, this does not mean just that there exists an $x y$ up-down path of length at least 4 , but also that there does not exist an xy up-down path of length at most 3.

Proof We employ the following distance arguments. 
- If $d_{N}(x, y)=2$, then $N$ must contain $\Lambda(x, y)$.

- If $d_{N}(x, y)=3$, then there is at most one reticulation on the shortest $x y$ up-down path. So if in addition we have that

- there are no reticulations on the shortest $x y$ up-down path. Then, $N$ must contain $\lambda(x, y)$ or $\lambda(y, x)$;

- there is one reticulation on the shortest $x y$ up-down path then $N$ must contain a reticulated cherry $K(x, y)$ or $K(y, x)$. If in addition we have that

- the parent of $x$ and the parent of $y$ share a common parent, then we say specifically that $N$ must contain $A(x, y)$ or $A(y, x)$;

- the parent of $x$ and the parent of $y$ do not share a common parent, then we say specifically that $N$ must contain $H(x, y)$ or $H(y, x)$.

- If $d_{N}(x, y) \geq 4$ then $N$ must contain $\Pi(x, y)$.

We now show that the shape on leaves $x$ and $y$ in a tree-child network is identifiable from the shapes on $x$ and $y$ in its MLLSs. This is summarized in Table 1. We start with the following theorem, which shows that each shape is preserved in at least one MLLS.

Theorem 4 Let $N$ be a level- $k$ tree-child network where $k \geq 2$, and let $x, y$ be two leaves in $N$. If $N$ contains $\Lambda(x, y), \lambda(x, y), A(x, y), H(x, y)$ or $\Pi(x, y)$, then there is an MLLS of $N$ containing $\Lambda(x, y), \lambda(x, y), A(x, y), H(x, y)$ or $\Pi(x, y)$, respectively.

Proof In this proof, we refer to the node labels used in Lemma 15.

The case that $N$ contains $\Lambda(x, y)$ is trivial.

Now suppose $N$ contains $\lambda(x, y)$. If $c$, the sibling of $y$, is a reticulation then deleting the reticulation edge leading into $c$ that is not $(b, c)$ returns an MLLS containing $\lambda(x, y)$. If $c$ is not a reticulation, then deleting any reticulation edge will not affect the shortest $x y$ up-down path. This results in an MLLS containing $\lambda(x, y)$.

Suppose $N$ contains $A(x, y)$. As $x, y$ are leaves, $A(x, y)$ is a level- $1 \mathrm{blob}$, and thus by definition, every MLLS of $N$ contains $A(x, y)$.

Suppose $N$ contains $H(x, y)$. If the blob containing the reticulation of $H(x, y)$ is of level lower than $k$, then every MLLS of $N$ contains $H(x, y)$, and we are done. So suppose this blob is level- $k$. As $k \geq 2$, there exists a reticulation $r$, which is not $c$, with reticulation edges $e$ and $f$. Let $N^{\prime}$ and $N^{\prime \prime}$ be the MLLSs of $N$ obtained by deleting $e$ and $f$ (among other reticulation edges), respectively. We claim that at least one of $N^{\prime}$ or $N^{\prime \prime}$ contains $H(x, y)$. Indeed, if $N^{\prime}$ contains $A(x, y)$, then in $N$, either $b$ or $i$ must be incident to $e$, as otherwise $a$ and $c$ will still have different parents after deleting $e$ and cleaning up. Now, $b$ cannot be incident to $e$ as it violates the tree-child property, regardless of whether $b$ is the tree node or the reticulation incident to $e$. Then, $i$ must be incident to $e$. If $i$ is $r$, then we note that $b$ cannot be the parent of $i$ due to the tree-child property. This implies that upon deleting $e$ and cleaning up, $a$ and $c$ have different parents, and subsequently $N^{\prime}$ contains $H(x, y)$. Thus, this case is impossible. If, on the other hand, $i$ is the tree node of $e$, then neither $i$ nor $b$ are suppressed after deleting $f$ and cleaning up. This implies that $N^{\prime \prime}$ contains $H(x, y)$. 
Suppose $N$ contains $\Pi(x, y)$. Suppose first that $d_{N}(x, y) \geq 5$. Take any $x y$ updown path in $N$, and consider $B T(N)$. Note that any up-down path in $N$ can be mapped to an up-down path in $B T(N)$. The 'up' portion of the path passes through the blob nodes containing $x$ in their label, until the first blob node containing $y$ is reached. The 'down' portion of the path passes through the blob nodes containing $y$ in their label, until a lowest blob node containing $y$ is reached. In particular, the apex is contained in the lowest blob which contains both $x$ and $y$ in their leaf-descendant set. So every $x y$ up-down path in $N$ passes through the same set of blobs $\mathcal{B}$. Furthermore, every $x y$ up-down paths enter and leave the blobs $B \in \mathcal{B}$ at the same nodes. Let $t_{B}$ and $h_{B}$ denote these nodes, respectively.

We claim that there is a reticulation edge we can delete from any blob $B \in \mathcal{B}$ of level- $k$ such that every $x y$ up-down path uses at least one edge from $B$ in the resultant subnetwork. We assume $l v l(B)=k$ as otherwise the claim holds trivially. At least one of $t_{B}$ or $h_{B}$ must be a reticulation, since we enter, pass through, and leave the blob $B$. We consider the cases when they are both reticulations and when $t_{B}$ is a reticulation but $h_{B}$ is not. Suppose first that $t_{B}$ and $h_{B}$ are both reticulations. Then $B$ must contain the apex of any $x y$ up-down path; furthermore, because of the tree-child property, the shortest $t_{B} h_{B}$ up-down distance must be at least 3 . Then, deleting a reticulation edge incident to $h_{B}$ either disconnects the $x y$ up-down path or reduces the length by at most 1 . In any case, at least one edge of $B$ is still used in the $x y$ up-down paths in the resultant subnetwork. Now suppose that $t_{B}$ is the only reticulation. Suppose $h_{B}$ is not incident to any reticulation edge. Since $l v l(B)=k \geq 2$, there exists a reticulation edge we can delete from $B$, such that neither $t_{B}, h_{B}$, nor the edge $\left(t_{B}, h_{B}\right)$ are suppressed. Now suppose $h_{B}$ is incident to a reticulation edge into a reticulation $r$. If this edge is also incident to $t_{B}$, then again since $\operatorname{lv} l(B)=k \geq 2$, there exists a reticulation edge we can delete from $B$, such that neither $t_{B}, h_{B}$, nor the edge $\left(t_{B}, h_{B}\right)$ are suppressed. Finally, if the edge is not incident to $t_{B}$, then deleting the reticulation edge incident to $r$ that is not $\left(h_{B}, r\right)$ ensures that $t_{B}, h_{B}$, nor $\left(t_{B}, h_{B}\right)$ are suppressed. In any case, deleting the chosen reticulation edge returns a subnetwork in which an edge of $B$ is used in every $x y$ up-down path.

So if $|\mathcal{B}| \geq 2$, then there exists an MLLS $N^{m l l s}$ in which all $x y$ up-down paths use at least two edges from the blobs in $\mathcal{B}$ plus at least three edges connecting the two blobs, $x$, and $y$. Therefore, $d_{N^{m l l s}}(x, y) \geq 5$. If $|\mathcal{B}|=1$, then by Lemma 14 , there exists an MLLS $N^{m l l s}$ with $d_{N^{m l l s}}(x, y) \geq 4$. Thus, if $d_{N}(x, y) \geq 5$, then there is an MLLS of $N$ containing $\Pi(x, y)$ (Fig. 11).

Suppose now that $d_{N}(x, y)=4$. We first show that there are at most 2 shortest $x y$ updown paths in $N$. Let $u, v$ be the parents of $x, y$, respectively. Then, any shortest $x y$ updown path is always of the form $(x, u),(u, w),(w, v),(v, y)$ (disregarding directions) where $w$ is some node in $N$, and one of $u, v, w$ is the apex of the shortest up-down path. Note that $u$ and $v$ are always included in any $x y$ up-down path, since they are the parents of $x$ and $y$, respectively. Therefore, having two shortest $x y$ up-down paths where $u$ and $v$ are the apex in each would create a cycle in $N$, contradicting the fact that $N$ is a phylogenetic network. Therefore, if $u$ is the apex of a shortest $x y$ up-down path in $N$, then there cannot be a shortest $x y$ up-down path where $v$ is the apex. There can be, however, a second shortest $x y$ up-down path in $N$ where $w$ is the apex. 


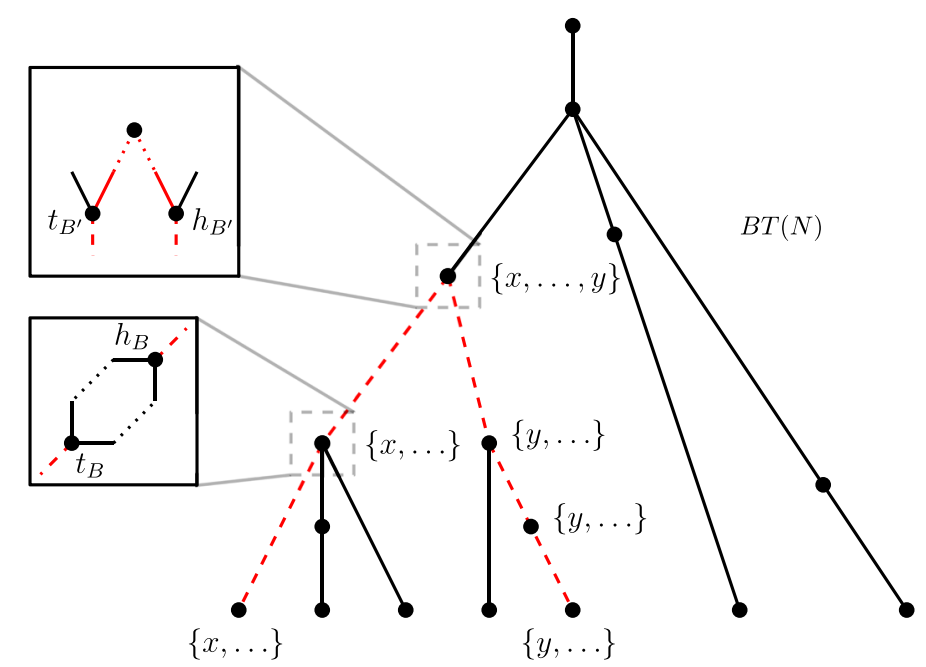

Fig. 11 (Color figure online) Proof visual of Theorem $4, d_{N}(x, y) \geq 5$ case. The red dashed up-down path in $B T(N)$ represents the trajectory of every $x y$ up-down path in $N$, and consequently, the set of blobs $\mathcal{B}$ through which every $x y$ up-down path passes. A zoomed-in portion of the two particular blob nodes illustrates the entry point $t_{B}$ and exit $h_{B}$ in $N$, and the case for when both points can be reticulations

Since $u, v$ are contained in all $x y$ up-down paths, we have that if two shortest $x y$ updown paths have the same apex, then they must be the same up-down paths. Otherwise the network would not be binary, or there would be parallel edges. If there were more than two shortest $x y$ up-down paths, then at least one of $u$ or $w$ would have degree greater than 3. This implies $N$ is non-binary, so there can be at most two shortest $x y$ up-down paths. This is shown in Fig. 12. Note that if there are two shortest $x y$ up-down paths in $N$, then it must be isomorphic to the one shown in Fig. 12, as otherwise the only other option would be to have $w$ and $w^{\prime}$ be the apex, in which case $w^{\prime}$ would be a parent of 2 reticulations, deeming $N$ to be not tree-child.

Now we show that if $d_{N}(x, y)=4$, then there is always an MLLS of $N$ containing $\Pi(x, y)$.

Suppose first that there are two shortest $x y$ up-down paths. Then, as stated before, it is isomorphic to the diagram shown in Fig. 12. There are no reticulation edges incident to either of the shortest paths other than on the reticulation at $v$. In particular, $(w, z)$ cannot be a reticulation edge because $N$ is tree-child. As $l v l(N) \geq 2$, there is another reticulation edge $e$ incident to a reticulation that is not $v$. Indeed, parents of $x$ and $y$ remain different and non-adjacent in the MLLS obtained by deleting $e$. This particular MLLS contains $\Pi(x, y)$.

Now suppose there is only one shortest $x y$ up-down path $P$. There are 5 nodes on $P$ including $x$ and $y$, and there are at most two reticulation edges incident to $P$ and at most one on $P$ by the tree-child property. Since $l v l(N) \geq 2$, there is at least one reticulation edge such that its deletion does not affect $P$. Deleting this reticulation edge and cleaning up ensures that the parents of $x$ and $y$ remain different and non-adjacent in the resultant MLLS. Therefore, there exists an MLLS of $N$ which contains $\Pi(x, y)$.

Thus, if $d_{N}(x, y)=4$, then there exists an MLLS of $N$ containing $\Pi(x, y)$. Therefore, if $N$ contains $\Pi(x, y)$, there exists an MLLS of $N$ containing $\Pi(x, y)$. 
Fig. 12 (Color figure online) An example of 2 shortest $x y$ up-down paths in $N$, whenever $d_{N}(x, y)=4$. One up-down path (red dashed) is $(x, u),(u, w),(w, v),(v, y)$ and the other (blue dashdotted) $(x, u),\left(u, w^{\prime}\right),\left(w^{\prime}, v\right),(v, y)$ (disregarding directions)

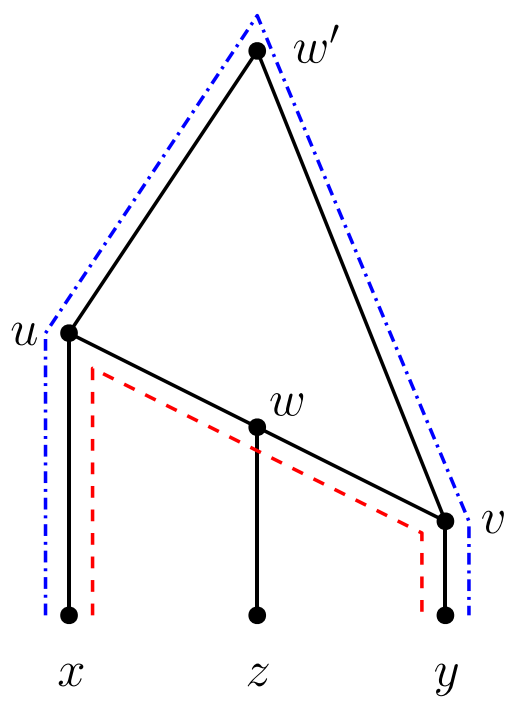

Lemma 16 For a tree-child network $N$, if $N$ contains $\Pi(x, y)$, then no MLLS of $N$ contains $\Lambda(x, y)$.

Proof We prove the contrapositive. Suppose one of the MLLSs $N^{m l l s}$ of $N$ contains $\Lambda(x, y)$. Add the deleted reticulation edges back to $N^{m l l s}$. Then, every node on a shortest $x y$ up-down path, excluding the apex and the leaves $x, y$, is incident to a reticulation edge. We first show that these nodes cannot be pure nodes in $N$.

Suppose for a contradiction that one of these nodes $p$ is a pure node in $N$. Then, $p$ must be a tree node, and there must exist two disjoint paths from $p$ to its reticulation child $r$. Without loss of generality, suppose that $p$ is above $x$. Since $p$ must be above $r$ via a path that does not use the edge $(p, r)$, there exists a node $z$ that is above $x$ and below $p$ such that $z$ is above $r$. When we delete the reticulation edges again to obtain $N^{m l l s}$, we must delete two edges from the blob with pure node $p$, which is impossible. We have a contradiction.

Now suppose for a contradiction that there are two nodes $u, v$ on a shortest $x y$ up-down path in $N$ excluding the apex. By our assumption, $u$ and $v$ are contained in a level- $k$ blob. By the above claim, neither $u$ nor $v$ can be pure nodes in $N$, and we note that the blob containing $u$ contains the apex, and the blob containing $v$ also contains the apex. This implies that $u$ and $v$ are contained in the same level- $k$ blob. To obtain $N^{m l l s}$, only one of $u$ or $v$ can be suppressed. In particular, $(u, v)$ cannot be an edge in $N$ as otherwise, this blob would be a level-1 blob. This implies that $N^{m l l s}$ does not contain $\Lambda(x, y)$, a contradiction.

Therefore, there can only be one node on a shortest $x y$ up-down path in $N$ excluding the apex, and thus $d_{N}(x, y) \leq 3$. Hence, $N$ does not contain $\Pi(x, y)$.

Theorem 5 Let $N$ be a level- $k$ tree-child network where $k \geq 2$, and let $x$, $y$ be two leaves in $N$. The shape on $\{x, y\}$ in $N$ is identifiable from the shapes on $\{x, y\}$ in the MLLSs. 
Proof We now prove a series of claims which state that $N$ contains a certain shape if and only if there are distinct MLLSs of $N$ containing certain shape(s) on $\{x, y\}$, and not containing certain other shape(s) on $\{x, y\}$.

Claim $1 N$ contains $\Lambda(x, y)$ if and only if all MLLSs of $N$ contain $\Lambda(x, y)$.

Proof 1 To show necessity, suppose $N$ contains $\Lambda(x, y)$ so that $d_{N}(x, y)=2$. Since the parent of $x$ and $y$ is a tree node, there is no reticulation edge incident to $\Lambda(x, y)$. Then, $\Lambda(x, y)$ is contained in every maximum subnetwork of $N$, and therefore in every MLLS of $N$.

For sufficiency, suppose for a contradiction that all MLLSs of $N$ on $X$ contain $\Lambda(x, y)$, but $N$ does not. If $N$ contains $\lambda(x, y), \lambda(y, x), K(x, y), K(y, x)$, or $\Pi(x, y)$ then, as these are the only possible shapes and their shapes are preserved in some MLLSs by Theorem 4, we have our required contradiction. Thus, the claim holds.

Claim $2 N$ contains $\lambda(x, y)$ if and only if there exists an MLLS of $N$ containing $\lambda(x, y)$ and no MLLSs of $N$ contain $\lambda(y, x), K(x, y), K(y, x)$ or $\Pi(x, y)$.

Proof 2 To show necessity note that by Theorem 4, there is an MLLS of $N$ that contains $\lambda(x, y)$. The only possible reticulation edge incident to $\lambda(x, y)$ is at $b$ whenever $c$ is a reticulation. Deleting the edge $(b, c)$ returns an MLLS containing $\Lambda(x, y)$, and deleting the reticulation edge incident to $c$ that is not $(b, c)$ returns an MLLS containing $\lambda(x, y)$. All other reticulation edges do not intersect $\lambda(x, y)$, and hence, their deletions do not affect $\lambda(x, y)$. Thus, an MLLS of $N$ does not contain $\lambda(y, x), K(x, y), K(y, x)$ nor $\Pi(x, y)$. The condition is therefore necessary.

To show sufficiency, suppose for a contradiction that the conditions hold but $N$ does not contain $\lambda(x, y)$. If $N$ contains $\Lambda(x, y)$ then by Claim 1 , no MLLSs of $N$ contain $\lambda(x, y)$, a contradiction. If $N$ contains $\lambda(y, x), K(x, y), K(y, x)$, or $\Pi(x, y)$, then as these are the only possible shapes and their shapes are preserved in some MLLSs by Theorem 4, we have our required contradiction. The condition is necessary, and the claim holds.

Since $A(x, y)$ is a level-1 blob in $N$ for two leaves $x, y \in X$, Claim 3 is trivially true.

Claim $3 N$ contains $A(x, y)$ if and only if all MLLSs of $N$ contain $A(x, y)$.

When $N$ contains $H(x, y)$, let $B_{H}$ be the blob containing the reticulation in $H(x, y)$.

Claim $4-N$ contains $H(x, y)$ and $\operatorname{lvl}\left(B_{H}\right)=k$ if and only if there exist distinct MLLSs of $N$ containing $\Lambda(x, y)$ and $H(x, y)$, and no MLLSs of $N$ contain $K(y, x)$.

$-N$ contains $H(x, y)$ and $\operatorname{lvl}\left(B_{H}\right)<k$ if and only if all MLLSs of $N$ contain $H(x, y)$.

Proof 4 We first prove the first statement of the claim. We first show necessity. Isolating $\langle x, y\rangle$ returns an MLLS of $N$ containing $\Lambda(x, y)$. By Theorem 4 , there is an 
MLLS of $N$ which contains $H(x, y)$. For the third condition, suppose for a contradiction that some MLLS $N^{m l l s}$ of $N$ contains $K(y, x)$. Since we have a reticulation on $y$ in $H(x, y)$, and because isolating $\langle x, y\rangle$ returns $\Lambda(x, y), N^{m l l s}$ must have been obtained by cutting $\langle x, y\rangle$. But then we have that the node $b$, the grandparent of $y$, has only reticulation children in $N$, contradicting the tree-child property of $N$. We therefore have necessity.

To show sufficiency, suppose for a contradiction that the conditions hold but $N$ does not contain $H(x, y)$. If $N$ contains $\Lambda(x, y)$ then by Claim 1 , no MLLSs of $N$ contain $H(x, y)$, a contradiction. If $N$ contains $\lambda(x, y)$ or $\lambda(y, x)$, then no MLLSs of $N$ contains $H(x, y)$ by Claim 2, a contradiction. If $N$ contains $A(x, y)$, then no MLLSs of $N$ contains $\Lambda(x, y)$ by Claim 3, a contradiction. If $N$ contains $K(y, x)$, then the shape is preserved in some MLLS of $N$ by Theorem 4, a contradiction. Finally, if $N$ contains $\Pi(x, y)$, then no MLLS of $N$ contains $\Lambda(x, y)$ by Lemma 16 , a contradiction. As these are the only possibilities, necessity follows. The claim holds for $\operatorname{lvl}\left(B_{H}\right)=k$.

We now prove the second statement of the claim. We first show necessity. Now suppose that $N$ contains $H(x, y)$ and $l v l\left(B_{H}\right)<k$. Then, none of the reticulation edges in $B_{H}$ are deleted to obtain any of the MLLSs of $N$ by definition. It follows that all MLLSs of $N$ contain $H(x, y)$.

We now show sufficiency. Suppose first that every MLLS of $N$ contains $H(x, y)$. If $N$ contained a shape that was not $H(x, y)$, then there exists an MLLS of $N$ that contains that particular shape by Theorem 4 . As this is a contradiction, we have that $N$ contains $H(x, y)$. To show that $l v l\left(B_{H}\right)<k$, we note that if this was not the case, i.e., if $l v l\left(B_{H}\right)=k$, then we have shown above that an MLLS of $N$ would contain $\Lambda(x, y)$, which is a contradiction. So we must have that $N$ contains $H(x, y)$ and that $l v l\left(B_{H}\right)<$ $k$.

Claim $5 N$ contains $\Pi(x, y)$ if and only if there exists an MLLS of $N$ containing $\Pi(x, y)$ and no MLLSs of $N$ contain $\Lambda(x, y)$.

Proof 5 We first show necessity. There is an MLLS of $N$ that contains $\Pi(x, y)$ by Theorem 4. By Lemma 16, no MLLSs of $N$ contains $\Lambda(x, y)$.

To show sufficiency, suppose for a contradiction that the conditions hold, but that $N$ does not contain $\Pi(x, y)$. If $N$ contains $\Lambda(x, y)$ then by Claim 1, every MLLS of $N$ contains $\Lambda(x, y)$, a contradiction. If $N$ contains $\lambda(x, y)$ or $\lambda(y, x)$ then no MLLSs of $N$ contain $\Pi(x, y)$ by Claim 2, a contradiction. If $N$ contains $A(x, y)$ or $A(y, x)$, then all MLLSs of $N$ contains $A(x, y)$ or $A(y, x)$ by Claim 3. This is a contradiction as no MLLSs of $N$ would contain $\Pi(x, y)$. If $N$ contains $H(x, y)$ or $H(y, x)$, then we split into two cases. Recall that $B_{H}$ is the blob of $N$ which contains $H(x, y)$ or $H(y, x)$. If $\operatorname{lvl}\left(B_{H}\right)<k$, then all MLLSs of $N$ contains $H(x, y)$ or $H(y, x)$ by Claim 4. This is a contradiction as no MLLSs of $N$ would contain $\Pi(x, y)$. If $\operatorname{lvl}\left(B_{H}\right)=k$, then there exists an MLLS of $N$ which contains $\Lambda(x, y)$ by Claim 4 , a contradiction. The condition is sufficient. The claim therefore holds.

Theorem 5 is summarized in Table 1. The table covers all of the different cases, showing which shapes can appear in MLLSs given the shape that the original network 


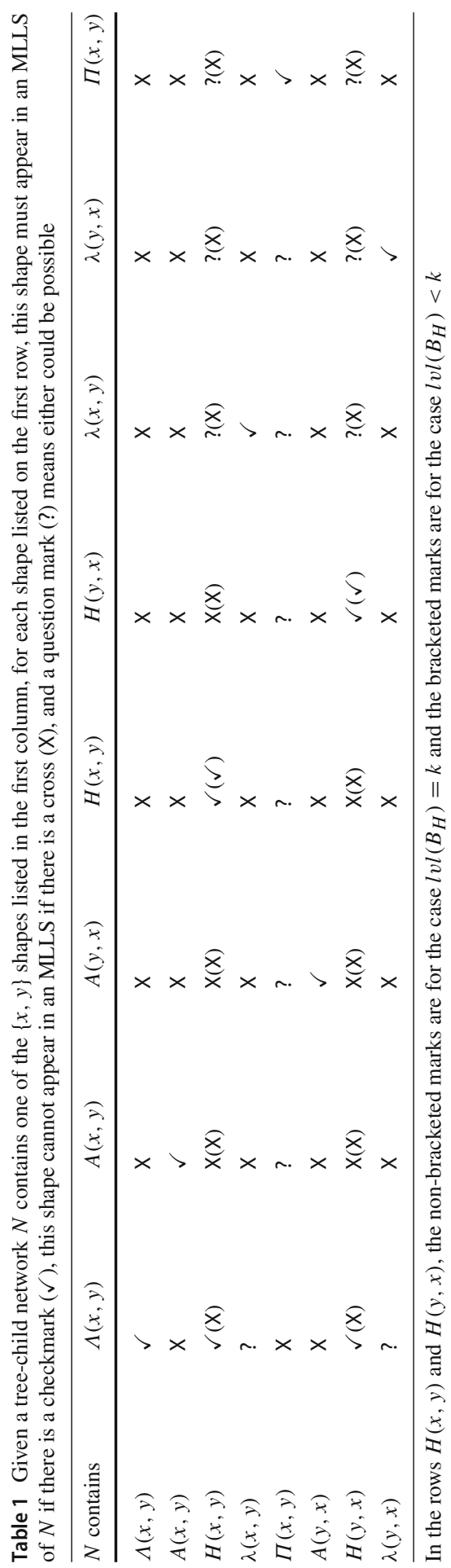




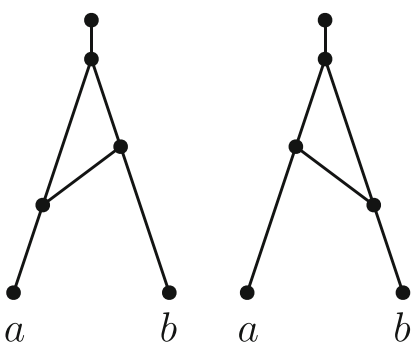

(a)

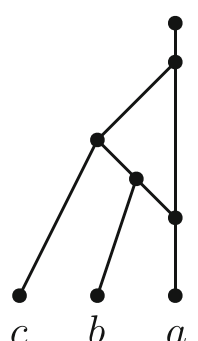

$c$

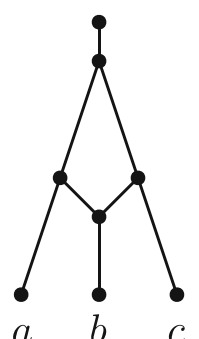

(b)

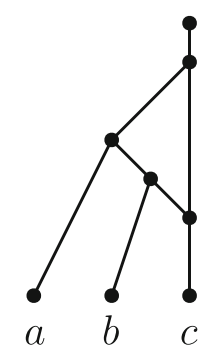

$c$

Fig. 13 a Two non-isomorphic level-1 networks with girth 3 that share the same subnetworks. b Three non-isomorphic level-1 networks with girth 4 that share the same subnetworks

contains. For any two rows in the table, there is some column in which one row has a check and the other a cross. Thus, we can distinguish between any two cases just by looking at the MLLSs, and so we can determine the structure between $x$ and $y$ on $N$. Because the given shapes are the only possibilities between two leaves $x$ and $y$, the table covers all possible cases.

\section{Reconstructibility of Tree-Child Networks}

In this section, we show that the class of tree-child networks, excluding trees and level1 networks with girth at most 4, is MLLS-reconstructible and thus level-reconstructible and subnetwork-reconstructible (where the girth is the length of a smallest cycle in the underlying undirected graph). A pair of level-1 networks with girth 3 and a triple of level-1 networks with girth 4 that is not subnetwork-reconstructible is shown in Fig. 13.

Following the leaf pair analysis in Sect. 4, we show here that it is possible to infer the location of a missing reticulation edge for level- $k$ blobs from the MLLSs. By Lemma 7, there exists a cherry or a reticulated cherry in every tree-child network. We know that the common parents within cherries are level- 0 blobs and $A$ shapes are level-1 blobs. Then, the reconstruction of level- $k$ blobs can be accomplished by reconstructing an $H$ shape of every level- $k$ blob.

We start by analyzing the possible shapes on $x, y$ after cutting a reticulated cherry on $x$ and $y$, see Fig. 14 for examples.

Lemma 17 Let $N$ be a tree-child network and suppose $N$ contains $H(x, y)$ on a leaf pair $\{x, y\}$. Then, the maximum subnetwork obtained by cutting the reticulated cherry $\langle x, y\rangle$ contains one of $\lambda(x, y), \lambda(y, x)$, or $\Pi(x, y)$. Furthermore, all other maximum subnetworks of $N$ contain either $\Lambda(x, y)$ or $H(x, y)$.

Proof Suppose for a contradiction that cutting $\langle x, y\rangle$ returns a maximum subnetwork $N^{\prime}$ of $N$ containing either $\Lambda(x, y), K(x, y)$, or $K(y, x)$. If $N^{\prime}$ contains $\Lambda(x, y)$, then the parent of $x$ and the parent of $y$ must share a common parent in $N$. This implies that $N$ contains $A(x, y)$, a contradiction. If $N^{\prime}$ contains $K(x, y)$, then the parent of $y$ is 


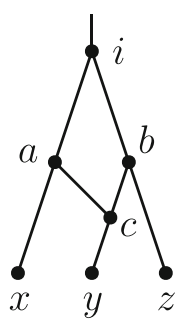

$$
\lambda(x, y)
$$

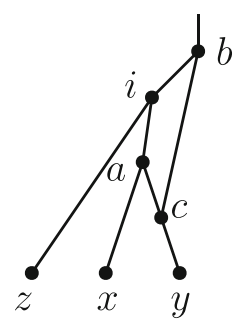

$\lambda(y, x)$

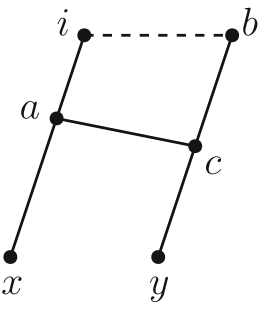

$\Pi(x, y)$

Fig. 14 The three cases for $H(x, y)$ in Lemma 17. Deleting edge $(a, c)$ yields $\lambda(x, y), \lambda(y, x)$, and $\Pi(x, y)$, respectively

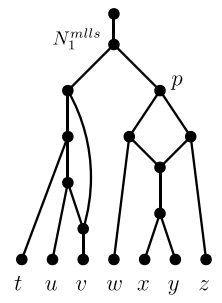

$N_{1}^{\max }$ contains $\Lambda(x, y)$

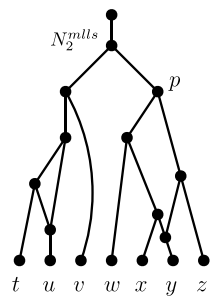

$N_{2}^{\max }$ contains $H(x, y)$

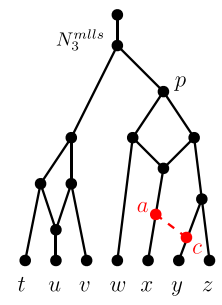

$N_{3}^{\max }$ contains $\Pi(x, y)$

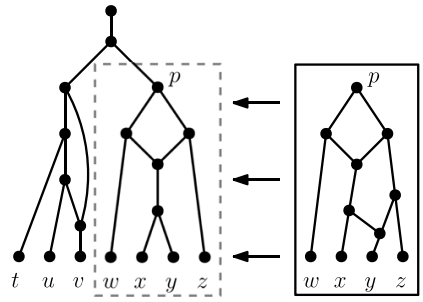

Reconstructing $B$ in $N_{1}^{\max }$

Fig. 15 (Color figure online) Three MLLSs $N_{1}^{m l l s}, N_{2}^{m l l s}$, and $N_{3}^{m l l s}$ of a level-2 tree-child network containing exactly two level-2 blobs. The three MLLSs contain $\Lambda(x, y), H(x, y)$, and $\Pi(x, y)$, respectively. We reconstruct the blob $B$ with pure node $p$ in $N_{3}^{\text {mlls }}$ initially and then reconstruct it in the other MLLSs. In $N_{3}^{m l l s}$, nodes $a, c$ are inserted directly above $x, y$, respectively, and an edge $(a, c)$ is added (red dashed edge). To reconstruct $B$ in $N_{1}^{m l l s}$ the pendant subnetwork rooted at $p$ is replaced by the reconstructed pendant subnetwork

a child of a reticulation in $N$. This contradicts the tree-child property of $N . N^{\prime}$ cannot contain $K(y, x)$ by Theorem 5 .

To prove the second statement of the lemma, note that isolating $H(x, y)$ returns a maximum subnetwork of $N$ that contains $\Lambda(x, y)$, and deleting any reticulation edge that is not incident to $y$ returns a maximum subnetwork that contains $H(x, y)$, since the parent of $x$ and the parent of $y$ is not suppressed and they are adjacent.

We now show how we can reconstruct a blob containing the reticulation of a reticulated cherry, see Fig. 15 for an example.

Lemma 18 Let $N$ be a level-k tree-child network, and suppose $N$ contains $H(x, y)$ for a leaf pair $\{x, y\}$. Suppose in addition that the blob $B$ containing the reticulation of $H(x, y)$ is level-k. Then, we can reconstruct $B$ in the MLLSs of $N$.

Proof By Theorem 5 and Lemma 17, $N$ contains $H(x, y)$ if and only if all MLLSs of $N$ contain either $\Lambda(x, y), H(x, y), \lambda(x, y), \lambda(y, x)$, or $\Pi(x, y)$, and there exist distinct MLLSs of $N$ that contain $\Lambda(x, y), H(x, y)$, and one of $\lambda(x, y), \lambda(y, x)$, or $\Pi(x, y)$. Now find the MLLS $N^{m l l s}$ of $N$ that contains one of $\lambda(x, y), \lambda(y, x)$, or $\Pi(x, y)$. Introduce nodes $a, c$ directly above $x, y$, respectively, and add an edge $(a, c)$ to $N^{m l l s}$. This reconstructs the blob $B$ in $N^{m l l s}$. 
It remains to show how to reconstruct $B$ in the other MLLSs. Hence, consider an arbitrary MLLS $N_{1}^{m l l s}$. Let $A \subseteq X$ denote the set of leaf-descendants of the pure node $p$ of $B$ in $N$. Then, $A$ is a node of $B T(N)$. Let $\Gamma(A)$ denote the set of all children of $A$ in $B T(N)$. Let $p_{i}$ for $i=1, \ldots,|\Gamma(A)|$ denote the corresponding pure nodes in $N$. In $N$, delete the tree edge leading into $p$, and also delete the two outgoing edges of $p_{i}$ for $i=1, \ldots,|\Gamma(A)|$, but do not clean up. Call the component that contains the node $p$ the $B$-part of $N$. By Theorem 2, the MLLS $N_{1}^{m l l s}$ contains a pure node $q$ with leaf-descendant set $A$ and pure nodes $q_{i}$ with leaf-descendant set equal to each set in $\Gamma(A)$.

In $N_{1}^{m l l s}$, delete the tree edge leading into $q$, and also delete the two outgoing edges of $q_{i}$, for $i=1, \ldots,|\Gamma(A)|$, but do not clean up. Call the component that contains the node $q$ the $B$-part of $N_{1}^{\text {mlls }}$. We can then reconstruct the blob $B$ in $N_{1}^{\text {mlls }}$ by replacing the $B$-part of $N_{1}^{m l l s}$ by the $B$-part of $N^{m l l s}$. Since $B$ is reconstructed correctly in $N^{m l l s}$, and since an edge deletion from a blob does not affect the network outside of the blob, it follows that this replacement correctly reconstructs the blob $B$ in $N_{1}^{m l l s}$.

Definition 11 Let $N$ be a tree-child network. A cherry $\Lambda(x, y)$ is reduced by deleting the node $y$ and cleaning up (same definition as in Bordewich and Semple 2016). A reticulated cherry $K(x, y)$ is reduced by isolating $K(x, y)$ and reducing the resultant cherry $\Lambda(x, y)$ (different definition to one in Bordewich and Semple 2016).

The following observation shows how we can obtain the MLLSs of a network obtained by reducing a reticulated cherry from the MLLSs of the original network. Note that a maximum subnetwork obtained by isolating a reticulated cherry in a treechild network remains tree-child by Lemma 3 and that a network obtained by reducing a cherry in a tree-child network also remains tree-child (Bordewich and Semple 2016).

Lemma 19 Let $N$ be a level-k tree-child network with a cherry or a reticulated cherry on a leaf pair $\{x, y\}$, and let $N^{\prime}$ be the tree-child network obtained by reducing $\{x, y\}$ from $N$.

- If $N$ contains $H(x, y)$ and the blob B containing $H(x, y)$ is oflevel- $k$, then, in each MLLS of $N$, reconstruct $B$ by Lemma 18 and subsequently reduce the reticulated cherry $H(x, y)$.

- Otherwise, reduce $\{x, y\}$ in all MLLSs of $N$.

Let $\mathcal{S}$ denote the set of networks we obtain from either of the above two cases. Then, $\mathcal{S}$ is precisely the set of all MLLSs of $N^{\prime}$.

Proof The MLLSs of $N^{\prime}$ are obtained by first reducing $\{x, y\}$ from $N$ and then finding the MLLSs of the resulting network. Since deleting edges from blobs has no effect on all other blobs in the network, we can in fact switch the order of reducing $\{x, y\}$ and deleting edges from level- $k$ blobs. In particular, the MLLSs of $N^{\prime}$ can also be obtained by deleting exactly one reticulation edge from all level- $k$ blobs of $N$ (that are not $B$, in the case that $N$ contains $H(x, y)$ and the blob $B$ containing $H(x, y)$ is of level- $k)$, and then subsequently reducing $\{x, y\}$ in all the resulting subnetworks. The latter process of obtaining the MLLSs of $N^{\prime}$ is exactly how the set $\mathcal{S}$ is obtained, and therefore, $\mathcal{S}$ is precisely the set of all MLLSs of $N^{\prime}$. 
Theorem 6 The class of binary level-k tree-child networks is MLLS-reconstructible, for $k \geq 2$.

Proof We prove by induction on $|X|$ that, for each level- $k$ tree-child network $N$ on $X$ with MLLS set $\mathcal{M}$, the network $N$ is the unique level- $k$ tree-child network with MLLS set $\mathcal{M}$. The base case $|X|=1$ is trivially true as when there is only one leaf, any network of level-2 or higher is no longer tree-child. So suppose $|X|>1$ and that the claim is true for each level- $k$ tree-child network on at most $|X|-1$ leaves. Let $N$ be a level- $k$ tree-child network on $X$ and let $\mathcal{M}$ be its MLLS set. We will show that the network $N$ can be reconstructed from $\mathcal{M}$, thus showing that $N$ is the unique network with MLLS set $\mathcal{M}$.

By Lemma 7, there exists at least one leaf pair $\{x, y\}$ that forms a cherry or a reticulated cherry in $N$.

If $N$ contains $H(x, y)$ and the blob $B$ containing the reticulation of $H(x, y)$ is of level- $k$, then correctly reconstruct $B$ in each element of $\mathcal{M}$ as outlined in Lemma 18. By Theorem 5, there is no other way of reconstructing the blob $B$. We update the elements of $\mathcal{M}$ by doing so. If $B$ is the only level- $k$ blob, we are done. Otherwise, we proceed as follows. Note that we can do this, as we can identify all level- $k$ blobs by Lemma 13.

At this point, all networks in $\mathcal{M}$ contain the same shape on $\{x, y\}$. Either all networks contain $\Lambda(x, y)$, all networks contain $A(x, y)$, or all networks contain $H(x, y)$.

Reduce $\{x, y\}$ in each network of $\mathcal{M}$, and call this new set of networks $\mathcal{S}$. Each network in $\mathcal{S}$ is tree-child and contains $|X|-1$ leaves. By Lemma $19, \mathcal{S}$ is the set of all MLLSs of $N^{\prime}$, the level- $k$ tree-child network obtained by reducing $\{x, y\}$ in $N$. By the induction hypothesis, $N^{\prime}$ is the unique level- $k$ tree-child networks with MLLS set $\mathcal{S}$. Reconstructing $N^{\prime}$ and undoing the reduction operation on $\{x, y\}$ yields the tree-child network $N$, which is therefore the unique level- $k$ tree-child network with MLLS set $\mathcal{M}$.

Gambette et al. have shown that level-1 networks with girth at least 5 are levelreconstructible (Gambette et al. 2017). The next corollary follows from their results, Observation 1, Theorem 6, and the following observation.

Observation 3 Let $N$ and $N^{\prime}$ be two tree-child networks that are both either level at least 2 or girth at least 4 . If the level of $N$ and $N^{\prime}$ is different, then they do not have the same set of lower-level subnetworks.

Corollary 3 The class of tree-child networks, excluding trees and level-1 networks with girth at most 4, is MLLS-reconstructible, level-reconstructible and subnetworkreconstructible.

\section{Reconstruction Algorithm for Tree-Child Networks}

In this section, we present an algorithm in the form of pseudo-code for reconstructing tree-child networks from their MLLSs. As shown in Sect. 5, we need only to reconstruct the $H$ shapes contained in level- $k$ blobs. Algorithm 1 systematically rebuilds every 
level- $k$ blob from the bottom-up, reducing common pendant subnetworks to leaves on the way. We give an example of Algorithm 1 in Fig. 16. To keep the description of the algorithm concise, it assumes that the input $\mathcal{T}$ consists of the set of MLLSs of some level- $k$ tree-child network, with $k \geq 2$. Nevertheless, the algorithm can in principle also be used to decide whether such a network exists or not. If the algorithm returns a network $N$, then we can check whether $\mathcal{T}=\mathcal{N}^{m l l s}(N)$. If this is not the case, or the algorithm fails to output a network, then such a network does not exist (see Theorem 7). Checking whether $\mathcal{T}=\mathcal{N}^{\text {mlls }}(N)$ can be done in $O\left(|\mathcal{T}|^{2}|X|^{2}\right)$ time, because checking whether two tree-child networks are isomorphic can be done in $O\left(|X|^{2}\right.$ ) time (Cardona et al. 2009).

Moreover, the algorithm can even be applied to an arbitrary set of level- $k-1$ treechild networks as input. If a network displaying the input networks exists the algorithm may find it, but is not guaranteed to do so (see Theorem 8.)

Before presenting the algorithm, we first go over a few key ideas required to prove the correctness and find the time complexity of the algorithm. We reiterate the idea of collapsing a pendant subnetwork from a network (presented in Sect. 3.3), and additionally define what it means to collapse a common pendant subnetwork from a set of networks. Let $N$ be a network and let $N_{A}$ be a pendant subnetwork of $N$ rooted by a node with leaf-descendant set $A$. Collapsing $N_{A}$ from $N$ means that we replace $N_{A}$ by a leaf $A$. Let $N \backslash N_{A}$ denote the network obtained by collapsing $N_{A}$ from $N$. Let $\mathcal{M}$ be a set of networks containing a common pendant subnetwork $N_{A}$. Collapsing $N_{A}$ from $\mathcal{M}$ means that we collapse $N_{A}$ from every network in $\mathcal{M}$. Let $\mathcal{M} \backslash N_{A}$ denote the set of networks obtained by collapsing $N_{A}$ from $\mathcal{M}$.

Lemma 20 Let $N$ be a level- $k$ tree-child network where $k \geq 2$. If there exists a common pendant subnetwork $N_{A}$ for the MLLSs of $N$, then $N_{A}$ is a pendant subnetwork of $N$.

Proof Consider the blob tree $B T\left(N_{A}\right)$. Since $N_{A}$ is a common pendant subnetwork of all MLLSs of $N$, the blob tree $B T\left(N_{A}\right)$ is a common pendant subtree of all blob trees of the MLLSs of $N$. By Theorem 2, BT $(N)$ must contain $B T\left(N_{A}\right)$. By Lemma 13, $N_{A}$ must be a level- $k^{\prime}<k$ network. This implies that no edge was deleted from $N_{A}$ in obtaining the MLLSs of $N$. Therefore, $N_{A}$ is a pendant subnetwork of $N$.

The following two observations follow directly from Lemma 13.

Observation 4 Let $N$ be a level-k tree-child network where $k \geq 2$. There exists no common pendant subnetwork for the MLLSs of $N$ if and only if all lowest blobs in $N$ are of level- $k$.

Observation 5 Let $N$ be a level-k tree-child network where $k \geq 2$. There exists $a$ common pendant subnetwork $N_{A}$ for the MLLSs of $N$ if and only if there exists a common pendant subtree rooted at A for the blob trees of the MLLSs of $N$.

Theorem 7 Let $N$ be a level- $k$ tree-child network on $X$ where $k \geq 2$, and let $\mathcal{T}=$ $\mathcal{N}^{\text {mlls }}(N)$. Algorithm 1 finds the network $N$ in time $O\left(|\mathcal{T}||X|^{3} / k\right)$.

Proof We first prove the correctness of the algorithm. By Lemma 20, every maximal common pendant subnetwork of $\mathcal{T}$ is a pendant subnetwork of $N$. Let $N_{A}$ be a maximal 


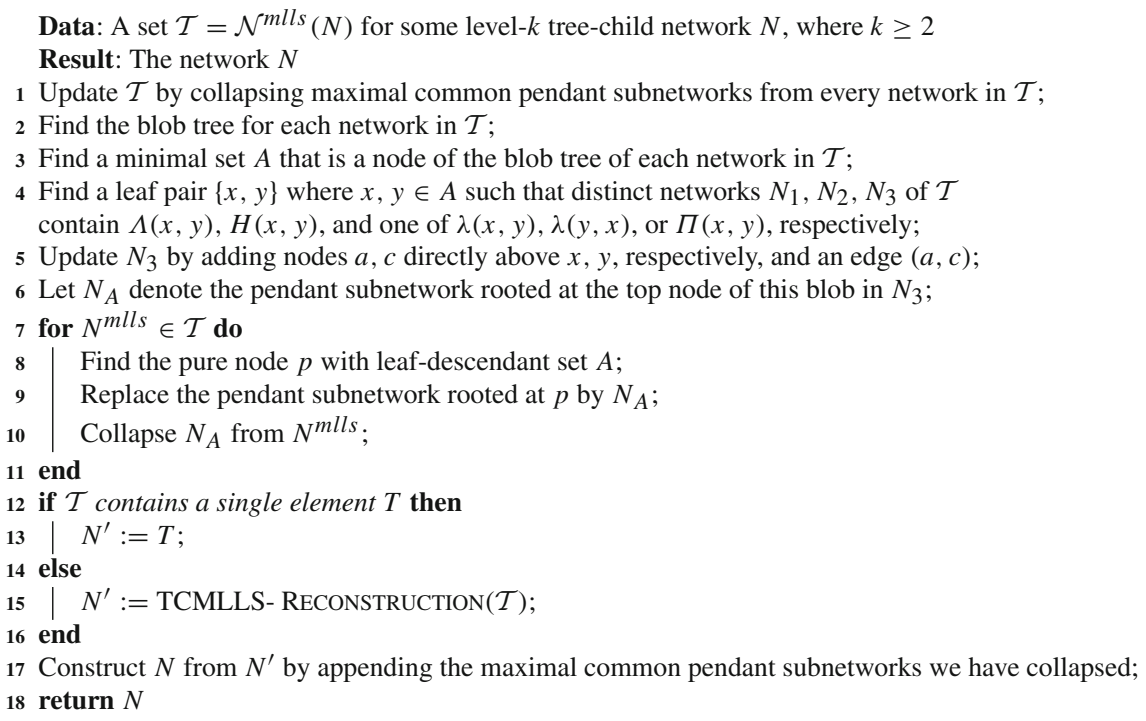

Algorithm 1: Algorithm TCMLLS- ReCOnSTRUCTION $(\mathcal{T})$

common pendant subnetwork of $\mathcal{T}$. Then, we can collapse $N_{A}$ from $N$ and $\mathcal{T}$, solve a smaller instance of the MLLS reconstruction problem by reconstructing $N \backslash N_{A}$ from $\mathcal{T} \backslash N_{A}$, and then appending $N_{A}$ to the leaf labeled $A$-which is the final step of the algorithm-returns the network $N$. Since all maximal pendant subnetworks are disjoint from one another, we can repeat this reduction for all maximal pendant subnetworks, by considering the reductions separately. Let $\mathcal{T}^{\prime}$ and $N^{\prime}$ denote the set of networks and network obtained by collapsing these pendant subnetworks from $\mathcal{T}$ and $N$, respectively. By Observation 4, we have that all lowest blobs of $N^{\prime}$ are of level$k$. We search for a lowest level- $k$ blob $B$ by finding a minimal set $A^{\prime}$ that is a node of the blob tree of each network in $\mathcal{T}$. Then, $A^{\prime}$ is a lowest foundation node of $N^{\prime}$ by Theorem 2. Then, we search for a leaf pair $\{x, y\}$ which form a reticulated cherry in $N^{\prime}$ with the reticulation in $B$. We note that such a leaf pair exists since $B$ is a lowest blob and since we have collapsed all common pendant subnetworks. Moreover, we can find it by searching for a pair of leaves as described in the algorithm in Table 1 and its proof in Theorem 5. Now we reconstruct $B$ using the steps outlined in the proof of Lemma 18. Let $A^{\prime}$ denote the leaf-descendant set of $B$, and let $N_{A^{\prime}}^{\prime}$ denote the corresponding pendant subnetwork (the subnetwork is pendant since $B$ is a lowest blob). At this point, if we only needed to reconstruct one level- $k$ blob (i.e., the case when $N^{\prime}$ contains one level- $k$ blob), then we have reconstructed $N^{\prime}$. Otherwise, collapsing $N_{A^{\prime}}^{\prime}$ gives the full set of MLLSs of the network $N^{\prime} \backslash N_{A^{\prime}}^{\prime}$. Continuing this reasoning, the recursive call will return the network $N^{\prime}$. Appending the collapsed maximum common pendant subnetworks to $N^{\prime}$ returns the network $N$.

Each recursive call of TCMLLS- RECONSTRUCTION reconstructs one level- $k$ blob, and therefore, the algorithm terminates once we have reconstructed all level- $k$ blobs, in which case we have reconstructed the network. 

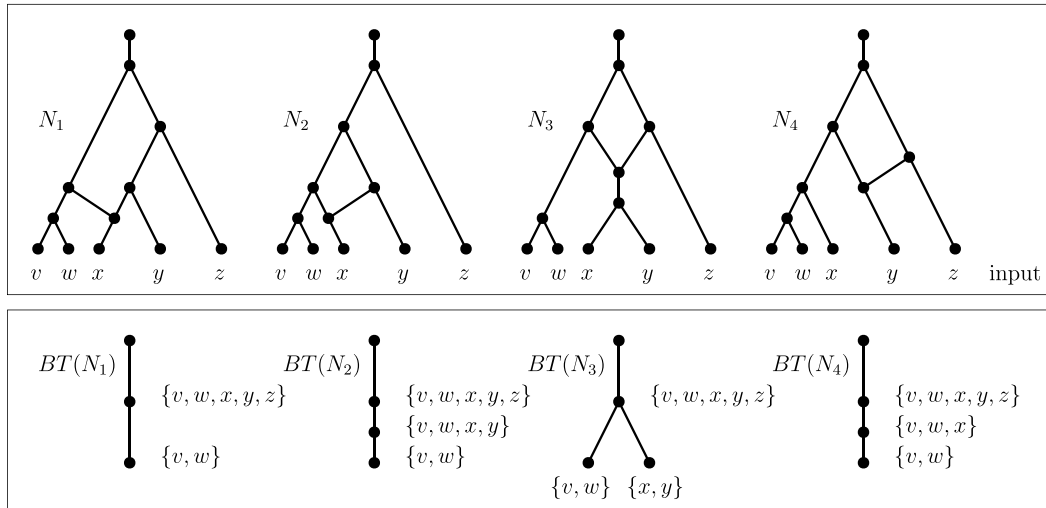

The set of foundation nodes is $\{\{v, w\},\{v, w, x, y, z\}\}$

The cherry $\Lambda(v, w)$ is the only common pendant subnetwork of the input networks. We reduce it to a leaf $\{v, w\}$.

Reducing common pendant subnetworks

In the reduced networks, we reconstruct the blob with leaf-descendant set $\{\{v, w\}, x, y, z\}$, since this set corresponds to a lowest foundation node.

$N_{1}$ contains $H(\{v, w\}, x)$

$N_{2}$ contains $H(\{v, w\}, x)$

$N_{3}$ contains $\Pi(\{v, w\}, x)$

$N_{4}$ contains $\Lambda(\{v, w\}, x)$

Then we add nodes $a, c$, and add edges $(a,\{v, w\}),(a, c),(c, x)$ to $N_{3}$

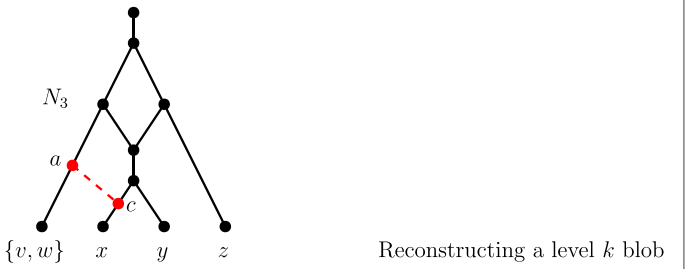

Upon reconstructing the same blob in $N_{1}, N_{2}$, and in $N_{4}$,

we note that all the updated networks are isomorphic.

We are in the if case of the algorithm, and thus it returns the above network, together with the cherry $\Lambda(x, y)$ appended to it.

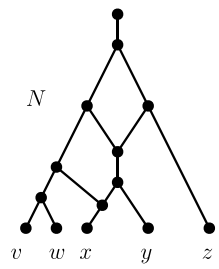

Fig. 16 (Color figure online) An example of the algorithm TCMLLS- RECONSTRUCTION $\left(\left\{N_{1}, N_{2}\right.\right.$, $\left.N_{3}, N_{4}\right\}$ ). Initially, the common pendant subnetworks of the four input networks are determined by looking at their blob trees. In this case, this is the cherry $\Lambda(v, w)$ (line 1). Upon reducing the cherry $\Lambda(v, w)$ to a leaf $\{v, w\}$ in all the MLLSs, the lowest foundation node is found to be $\{\{v, w\}, x, y, z\}$. We find a leaf pair $\{\{v, w\}, x\}$ specified in line 4 of the algorithm. Since $N_{3}$ contains $\Pi(\{v, w\}, x)$, we reconstruct this blob in $N_{3}$, shown by the red dashed edge (line 5). After reconstructing the same blob in all the other networks $N_{1}, N_{2}$ and $N_{4}$, we see that the networks are all isomorphic (we enter the if statement of line 12). The algorithm then returns the network $N$ 
For the running time, observe that each recursive call of TCMLLS- RECONSTRUCTION acts on an instance $\mathcal{N}^{m l l s}\left(N^{\prime}\right)$ on leaf set $X^{\prime}$ such that $\left|X^{\prime}\right|<|X|$ and there is one fewer level- $k$ blob that needs to be reconstructed in the networks of $\mathcal{N}^{m l l s}\left(N^{\prime}\right)$ when compared to that of $\mathcal{N}^{m l l s}(N)$. Since every level- $k$ blob has at least $k+1$ outgoing edges, the number of level- $k$ blobs in $N$ is at most $|X| / k$. Then, TCMLLS- RECONSTRUCTION is called at most $|X| / k$ times.

Each single iteration of TCMLLS- RECONSTRUCTION can be split into four partscollapsing largest common pendant subnetworks from the networks (lines 1-2), finding a lowest foundation node $A$ (i.e., finding a lowest level- $k$ blob) of $N$ (line 3), reconstructing a lowest level- $k$ blob (lines 4-11), and checking whether all updated networks are isomorphic to one another (line 12). Finding a largest common pendant subnetwork can be done by looking at the blob trees, which can all be constructed in $O(|\mathcal{T}||X|)$ time. By Observation 5, there exists a common pendant subnetwork rooted at the pure node with leaf-descendant set $A$, if there exists a common pendant subtree rooted at $A$ in the blob trees. The number of blob nodes is maximized for a tree on $|X|$ leaves (or whenever every reticulation is in a triangle), in which case it contains $2|X|$ nodes in total (including the root). Then, there exist at most $|X|-1$ foundation nodes, and thus, at most $|X|-1$ pendant subnetworks. Collapsing a pendant subnetwork from a network takes constant time, and for every possible pendant subnetwork, we iterate through the networks in $\mathcal{T}$. This step takes $O(|\mathcal{T}||X|)$ time. Finding a lowest foundation node follows immediately as we have found all pendant subnetworks of the blob trees. Then, this step takes constant time. The level- $k$ blob reconstruction chooses a pair of leaves which descend from a blob and subsequently searches through all networks in $\mathcal{T}$ to see if the pair is the $H$ shape we seek. This takes $O\left(|\mathcal{T}||X|^{2}\right)$ time if we try each pair of leaves (or $O\left(|\mathcal{T}|^{2}|X|\right)$ time if we try each cherry of each network). Collapsing pendant subnetworks take constant time, and we do this for every network in $\mathcal{T}$. Therefore, the running time for reconstructing a lowest level- $k$ blob still takes $O\left(|\mathcal{T}||X|^{2}\right)$ time (or $O\left(|\mathcal{T}|^{2}|X|\right)$ time). To decide whether all networks have become isomorphic, we only need to check whether each blob tree consists of just two nodes (including the root). It is not necessary to check whether some networks have become isomorphic after each recursion, since the algorithm still works if the input set contains isomorphic networks. The algorithm terminates when all level- $k$ blobs have been reconstructed: this is precisely when all networks become isomorphic.

Since we can construct (or update) the blob trees in $O(|\mathcal{T}||X|)$ time, we can decide whether all networks have become isomorphic in $O(|\mathcal{T}||X|)$ time.

Thus, the total time over a single iteration of TCMLLS-RECONSTRUCTION is $O\left(|\mathcal{T}||X|^{2}\right)$ (or $\left.O\left(|\mathcal{T}|^{2}|X|\right)\right)$.

It follows that the total running time of the algorithm is $O\left(|\mathcal{T}||X|^{3} / k\right)$ (or $\left.O\left(|\mathcal{T}|^{2}|X|^{2} / k\right)\right)$.

Here, we restrict the input data $\mathcal{T}$ to be the full set of MLLSs of some tree-child network $N$, and return $N$. We now show that it is not necessary to have this restriction: in fact, we require only three MLLSs to reconstruct $N$. That is, the same three MLLSs can be used to reconstruct each level- $k$ blob. However, if we do not have all MLLSs, we are unable to identify the level- $k$ blobs. Therefore, we require here that also the number of reticulations in the network is given. 
Theorem 8 Three MLLSs suffice to reconstruct a level-k tree-child network, with $k \geq$ 2, if the number $l$ of level-k blobs is known.

Proof Let $N$ be a level- $k$ tree-child network with $l$ level- $k$ blobs and $k \geq 2$. We first pick three MLLSs $N_{1}^{m l l s}, N_{2}^{m l l s}$, and $N_{3}^{m l l s}$ of $N$ and then show that Algorithm 1 returns $N$ with these inputs and that no other tree-child network exists with these three MLLSs and $l$ level- $k$ blobs.

Let $B_{1}, \ldots, B_{l}$ denote the level- $k$ blobs in $N$, and let, for $i=1, \ldots, l, r_{i}$ denote a reticulation in $B_{i}$ that is in a lowest reticulated cherry shape, which exists by Lemma 6. Since $B_{i}$ is a level- $k$ blob where $k \geq 2$, the parents of $r_{i}$ must be non-adjacent (otherwise $B_{i}$ would be a level-1 blob). Let $N_{1}^{\text {mlls }}$ denote the MLLS of $N$ obtained by cutting the reticulated cherry shapes that contain $r_{i}$, and let $N_{2}^{m l l s}$ denote the MLLS of $N$ obtained by isolating the reticulated cherry shapes that contain $r_{i}$. Let $N_{3}^{m l l s}$ denote the MLLS of $N$ obtained by deleting, from each level- $k$ blob $B_{i}$, a reticulation edge that is not incident to $r_{i}$, and such that the parents of $r_{i}$ remain non-adjacent in $N_{3}^{m l l s}$. To see that such an MLLS exists, recall that by Theorem 4, we have that if a network contains $H(x, y)$, then there is an MLLS of $N$ that contains $H(x, y)$. Therefore, by treating each level- $k$ blob $B_{i}$ as a level- $k$ tree-child network, we may invoke Theorem 4 to claim that such an MLLS $N_{3}^{\text {mlls }}$ exists.

Now we show that these three MLLSs suffice to reconstruct $N$ with Algorithm 1. Recall that Algorithm 1 initially collapses all maximal common pendant subnetworks from the input. Let $N^{\prime}$ denote the network obtained by collapsing the same pendant subnetworks from $N$. Then, the algorithm finds a minimal set $A$ that is a node of the blob tree of each of $N_{1}^{m l l s}, N_{2}^{m l l s}, N_{3}^{m l l s}$. By Lemma 12, A is a leaf of the blob tree of $N^{\prime}$. Since the MLLSs $N_{1}^{m l l s}, N_{2}^{m l l s}, N_{3}^{m l l s}$ were constructed in such a way that all common pendant subnetworks are of level strictly lower than $k$, the set $A$ is the set of leaf-descendants of some lowest level- $k$ blob of $N^{\prime}$. Note that, in $N^{\prime}, r_{1}$ is contained in a reticulated cherry $H(x, y)$ for some leaves $x, y$, since there are no blobs below $B_{1}$. By construction, the MLLS $N_{1}^{\text {mlls }}$ contains one of $\lambda(x, y), \lambda(y, x)$, or $\Pi(x, y)$. The MLLS $N_{2}^{m l l s}$ contains $\Lambda(x, y)$, and the MLLS $N_{3}^{m l l s}$ contains $H(x, y)$. Hence, we can argue similarly to in the proof of Theorem 7 that the algorithm then reconstructs the blob $B_{1}$ in all input MLLSs, and that recursing the algorithm reconstructs the next lowest level- $k$ blob (which can be reconstructed analogously as done for $B_{1}$ ). It follows then that the algorithm reconstructs $N$ after $l$ recursions of the algorithm.

We now show that no other tree-child level- $k$ network with $l$ level- $k$ blobs exists that displays $N_{1}^{m l l s}, N_{2}^{m l l s}$ and $N_{3}^{m l l s}$. Suppose such a network $N^{*}$ exists. Then, its MLLS set contains $N_{1}^{m l l s}, N_{2}^{\text {mlls }}$ and $N_{3}^{\text {mlls }}$. Hence, by the arguments above, running Algorithm 1 on the full set $\mathcal{N}^{m l l s}\left(N^{*}\right)$ of MLLSs of $N^{*}$ returns $N$. In particular, note that since $N^{*}$ has the same number of level- $k$ blobs as $N$, the same common pendant subnetworks are collapsed (in each recursive call) when running the algorithm on $\mathcal{N}^{m l l s}\left(N^{*}\right)$ as when we run the algorithm on $N_{1}^{m l l s}, N_{2}^{m l l s}$ and $N_{3}^{m l l s}$. By Theorem 7, running the algorithm on $\mathcal{N}^{m l l s}\left(N^{*}\right)$ returns $N^{*}$. Hence, $N^{*}$ and $N$ are isomorphic.

Note that in the proof of Theorem 8, we crafted three particular MLLSs $N_{1}^{m l l s}, N_{2}^{m l l s}$, and $N_{3}^{m l l s}$ however, there could be many triples of MLLSs which are sufficient in recon- 
structing the original network. Suppose that we have deleted the reticulation edge $e_{i}^{j}$ from the blob $B_{i}$ to obtain the MLLS $N_{j}^{m l l s}$ for $j=1,2,3$. The proof of Theorem 8 depends on the argument that if for every level- $k$ blob, we can find the particular three shapes, then we can reconstruct said level- $k$ blob. Then, we can define three new MLLSs that are also sufficient for reconstructing $N$ as follows. Let $N_{1}$ be the MLLS obtained by deleting one of the three reticulation edges $\left(e_{1}^{j}, e_{2}^{j}, e_{3}^{j}\right)$ from each level- $k$ blob $\left(B_{i}\right)$. Let $N_{2}$ be the MLLS obtained by deleting one of the remaining two reticulation edges, and let $N_{3}$ denote the MLLS obtained by deleting the remaining reticulation edges from $N$. If there were $l$ level- $k$ blobs in $N$, then we would have $3 l$ possible choices of $N_{1}, 2 l$ possible choices of $N_{2}$, and 1 possible choice of $N_{3}$. Therefore, we have $6 l^{2}$ possible choices for having a triple of MLLSs that are sufficient for reconstructing the network, given the reticulation edges $e_{i}^{j}$. Note that we simply looked at a particular lowest reticulation $r_{i}$ for each level- $k$ blob $B_{i}$ and also note that there could be more than one reticulation edge that we could have deleted in retrieving the MLLS $N_{3}^{m l l s}$ (in the proof of Theorem 8). This implies that there could be many more triples of MLLSs that suffice to reconstruct $N$.

Therefore, it is possible to reconstruct the network from a subset of the MLLSs, given that they hold enough information. In particular, if we have the three MLLSs as stated in the proof of Theorem 8, then our algorithm returns the network in time $O\left(|X|^{2} / k\right)$.

\section{Conclusion and Outlook}

In this paper, we have shown that level- $k$ tree-child networks, where $k \geq 2$, are determined by their MLLSs and provided a polynomial-time algorithm for reconstructing such a network from its MLLSs. We achieved this result by exploiting one of the tree-child properties - the lowest tree node is in a cherry or a reticulated cherry.

An apparent hindrance to our method is that there is no guarantee nor reason to have the set of all MLLSs of the original network. Converting sequence data into the MLLSs could be quite challenging, especially for higher level. It would therefore be interesting to focus on ways to make our results more practical, possibly employing similar approaches used in methods such as trinet-based methods (Oldman et al. 2016), which work with subnetworks with only three leaves.

On the positive side, we have shown that it is not necessary to know all MLLSs to reconstruct the original network: we need only three, see Theorem 8 . Therefore, a more plausible application of our approach would be the following. Suppose, for example, that different studies each manage to produce a network with some reticulations. However, in each of these networks some actual reticulate events have been missed, possibly due to computational limitations or lack of data. Then, a method based on our theoretical results could be used to reconstruct the full network from the networks with missing reticulate events.

Extending our MLLS reconstructibility results to a more general class of networks is another natural step forward. We briefly discuss and explain how it might be possible 

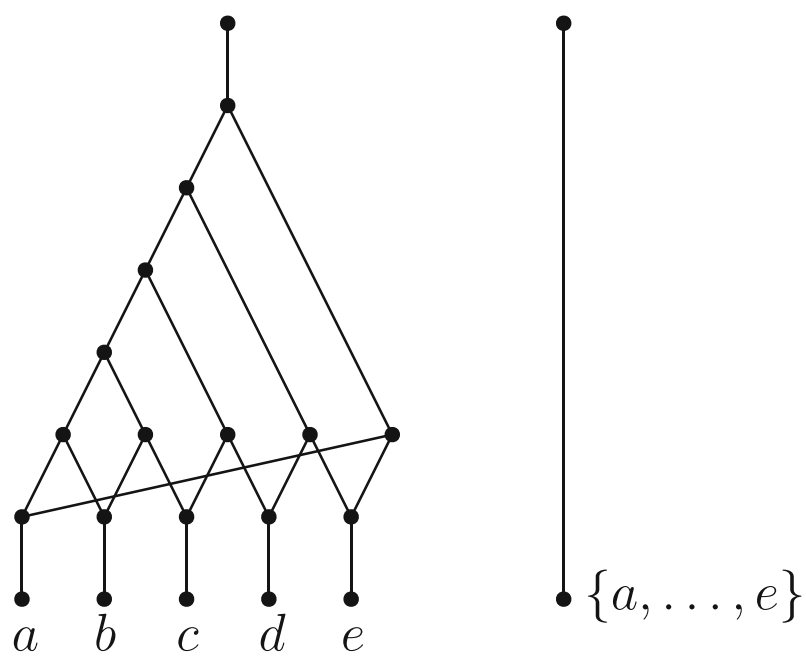

Fig. 17 A valid network with a tessellating crown blob and its blob tree. Deleting any of the reticulation edges keeps the original blob biconnected, and hence, it will not affect the blob tree

to adapt our results to the class of valid networks, where every reticulation edge in the network is valid.

In the case of valid networks, it is not always true that there exists a reticulation edge in every blob whose deletion results in a maximum subnetwork where the blob tree differs from the blob tree of the original network (i.e., an analogous statement to Observation 2 does not always hold for valid networks). An example of this, a tessellating crown, is shown in Fig. 17.

For leaf pair analysis, there is an extra shape on two leaves $\{x, y\}$ where both parents $p_{x}, p_{y}$ of $x, y$, respectively, are reticulations, and they share a common parent $g_{x}$. This shape, called a 2-reticulated cherry (see leaves $b, c$ of Fig. 17, defined in Bordewich et al. 2018a), is distinct from all others (given we adapt the definition of when $N$ contains $\Pi(x, y))$ as it contains $K(x, y)$ and $K(y, x)$ in its MLLSs, respectively. Unlike tree-child networks, MLLSs of valid networks can contain a cherry, reticulated cherry, or a 2-reticulated cherry stemming from the lowest tree node. The reconstruction of a 2-reticulated cherry poses a challenge as there are two potential places to reinsert the reticulation edge. That is, given an MLLS where $\left(g_{x}, p_{x}\right)$ has been deleted, we add two nodes $a, b$ with edge $(a, b)$. We know that $b$ must be placed directly above $x$. However, we have two possibilities for inserting $a$ above $p_{y}$ (illustrated in Fig. 18).

Nevertheless, we conjecture the following:

Conjecture 1 The class of binary valid networks is MLLS-reconstructible.

Note here that invalid networks, where not every reticulation edge is valid, are not level-reconstructible in general. A counter example is given in Fig. 19. 


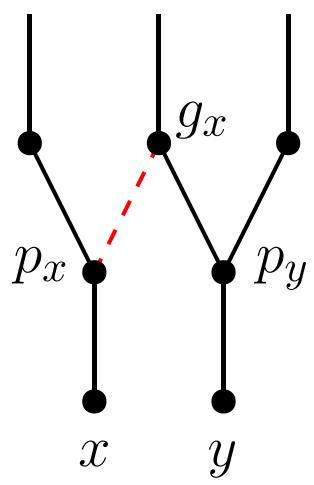

(a)

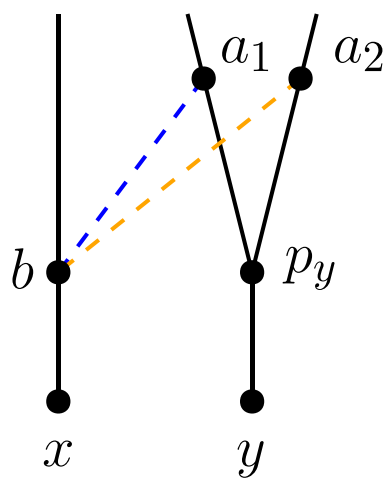

(b)

Fig. 18 (Color figure online) a 2-reticulated cherry on $\{x, y\}$. b MLLS where the red dashed edge is deleted from $\mathbf{a}$. We have two options, $a_{1}, a_{2}$, for inserting the reticulation edge
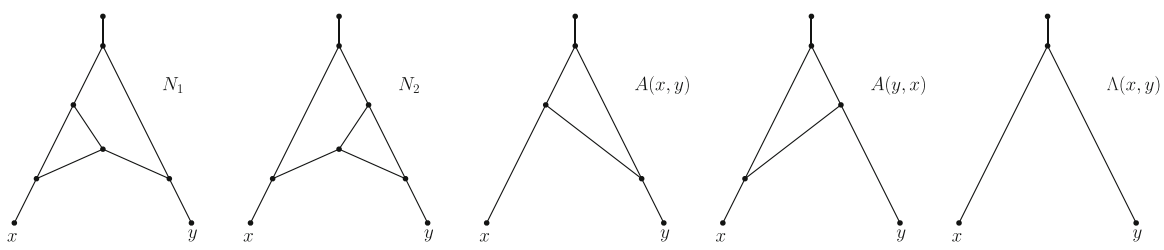

Fig. 19 Two level-2 networks $N_{1}$ and $N_{2}$ are non-isomorphic but have the same lower-level subnetworks. In general, invalid networks are not level-reconstructible

Ultimately, we wish to characterize precisely which networks are reconstructible from their MLLSs. Though this could perhaps be possible by an analogous leaf pair analysis as done in Sect. 4, we quickly reach a large number of cases, with level-2 networks already containing 15 possible shapes. A more efficient methodology will be required to treat such general networks. Considering the level- $k$ generators (van Iersel et al. 2009) may perhaps provide an interesting approach to this problem.

Acknowledgements We thank the anonymous referee for their helpful comments in improving this manuscript. Yukihiro Murakami, Leo van Iersel, Remie Janssen, and Mark Jones were supported in part by the Netherlands Organization for Scientific Research (NWO), including Vidi Grant 639.072.602, and Leo van Iersel also partly by the 4TU Applied Mathematics Institute. Vincent Moulton thanks the Netherlands Organization for Scientific Research (NWO) Vidi grant 639.072.602, for its support to visit TU Delft.

Open Access This article is distributed under the terms of the Creative Commons Attribution 4.0 International License (http://creativecommons.org/licenses/by/4.0/), which permits unrestricted use, distribution, and reproduction in any medium, provided you give appropriate credit to the original author(s) and the source, provide a link to the Creative Commons license, and indicate if changes were made.

\section{References}

Bordewich M, Semple C (2016) Determining phylogenetic networks from inter-taxa distances. J Math Biol 73(2):283-303 
Bordewich M, Semple C, Tokac N (2018a) Constructing tree-child networks from distance matrices. Algorithmica 80(8):2240-2259. https://doi.org/10.1007/s00453-017-0320-6

Bordewich M, Huber KT, Moulton V, Semple C (2018b) Recovering normal networks from shortest intertaxa distance information. J Math Biol 77:1-24

Cardona G, Rossello F, Valiente G (2009) Comparison of tree-child phylogenetic networks. IEEE/ACM Trans Comput Biol Bioinform 6(4):552-569

Gambette P, Huber KT, Kelk S (2017) On the challenge of reconstructing level-1 phylogenetic networks from triplets and clusters. J Math Biol 74(7):1729-1751

Gusfield D, Bansal V (2005) A fundamental decomposition theory for phylogenetic networks and incompatible characters. In: Annual international conference on research in computational molecular biology. Springer, pp 217-232

Hein J (1990) Reconstructing evolution of sequences subject to recombination using parsimony. Math Biosci 98(2):185-200

Huber KT, Moulton V (2013) Encoding and constructing 1-nested phylogenetic networks with trinets. Algorithmica 66(3):714-738

Huber KT, van Iersel L, Moulton V, Wu T (2014) How much information is needed to infer reticulate evolutionary histories? Syst Biol 64(1):102-111

Huson DH, Rupp R, Scornavacca C (2010) Phylogenetic networks: concepts, algorithms and applications. Cambridge University Press, Cambridge

Huynh TN, Jansson J, Nguyen NB, Sung WK (2005) Constructing a smallest refining galled phylogenetic network. In: Annual international conference on research in computational molecular biology. Springer, pp 265-280

Jansson J, Sung WK (2006) Inferring a level-1 phylogenetic network from a dense set of rooted triplets. Theor Comput Sci 363(1):60-68

Jin G, Nakhleh L, Snir S, Tuller T (2006) Maximum likelihood of phylogenetic networks. Bioinformatics 22(21):2604-2611

Morrison DA (2005) Networks in phylogenetic analysis: new tools for population biology. Int J Parasitol 35(5):567-582

Nakhleh L, Warnow T, Linder CR, John KS (2005) Reconstructing reticulate evolution in species-theory and practice. J Comput Biol 12(6):796-811

Oldman J, Wu T, van Iersel L, Moulton V (2016) Trilonet: piecing together small networks to reconstruct reticulate evolutionary histories. Mol Biol Evol 33(8):2151-2162

Pardi F, Scornavacca C (2015) Reconstructible phylogenetic networks: do not distinguish the indistinguishable. PLoS Comput Biol 11(4):e1004135

Sneath PH (1975) Cladistic representation of reticulate evolution. Syst Zool 24(3):360-368

Strimmer K, Moulton V (2000) Likelihood analysis of phylogenetic networks using directed graphical models. Mol Biol Evol 17(6):875-881

van Iersel L, Moulton V (2014) Trinets encode tree-child and level-2 phylogenetic networks. J Math Biol 68(7):1707-1729

van Iersel L, Keijsper J, Kelk S, Stougie L, Hagen F, Boekhout T (2009) Constructing level-2 phylogenetic networks from triplets. IEEE/ACM Trans Comput Biol Bioinform 6(4):667-681

van Iersel L, Moulton V, de Swart E, Wu T (2017) Binets: fundamental building blocks for phylogenetic networks. Bull Math Biol 79(5):1135-1154

von Haeseler A, Churchill GA (1993) Network models for sequence evolution. J Mol Evol 37(1):77-85

Willson SJ (2010) Properties of normal phylogenetic networks. Bull Math Biol 72(2):340-358

Willson SJ (2011) Regular networks can be uniquely constructed from their trees. IEEE/ACM Trans Comput Biol Bioinform 8(3):785-796

Publisher's Note Springer Nature remains neutral with regard to jurisdictional claims in published maps and institutional affiliations. 